\title{
Simulations of systematic direction-dependent instrumental effects in intensity mapping experiments
}

\author{
T. Ansah-Narh, ${ }^{1,6 \star}$ F. B. Abdalla, ${ }^{1,2}$ O. M. Smirnov, ${ }^{1,3}$ K. M. B. Asad ${ }^{1,3,4}$ and \\ J. R. Shaw ${ }^{5}$ \\ ${ }^{1}$ Department of Physics and Electronics, Rhodes University, PO Box 94, Grahamstown 6140, South Africa \\ ${ }^{2}$ Department of Physics and Astronomy, University College London, London WC1E 6BT, UK \\ ${ }^{3}$ SKA South Africa, 3rd Floor, The Park, Park Road, Pinelands 7405, South Africa \\ ${ }^{4}$ Department of Physics and Astronomy, University of the Western Cape, Bellville, Cape Town 7535, South Africa \\ ${ }^{5}$ Department of Physics and Astronomy, University of British Columbia, 6224 Agricultural Road, Vancouver V6T 1Z1, Canada \\ ${ }^{6}$ Ghana Space Science and Technology Institute, Ghana Atomic Energy Commission, P. O. Box LG 80, Legon, Ghana
}

Accepted 2018 August 25. Received 2018 August 25; in original form 2017 October 12

\begin{abstract}
Intensity mapping experiment treats the $21 \mathrm{~cm}$ radio emission as a diffuse source and allows smaller and relatively cheaper radio antennas with short baselines to be used in such experiments. However, the technique is restricted by the precise subtraction of the foreground continuum signal from Galactic and extragalactic radio sources. Furthermore, the signal is subjected to direction-dependent effects, particularly the primary beam, as it modulates the intensity as a function of the sky position. In addition, due to the imperfections in the antenna feeds, a portion of the polarized foreground tends to find its way into the total intensity, making it a major obstacle to detect the $\mathrm{H}$ I signal. In the case of dish arrays, this will be dominated by the instrument mispointings and polarization leakage. To estimate this contamination, we use OSKAR to simulate 'dish-like' primary beams and then perturb these primary beams by introducing gain, phase, and surface distribution errors. We then simulate the foregrounds with these modelled beams to determine the errors in Stokes $I$ and also observe the amount of $\mid Q$ $+i U \mid$ that corrupts $I$. Our simulation shows that the $\mathrm{H}$ I signal power can be measured at a multipole moment of $l=100$ if we do not correct for any polarization leakage of the beam and at a multiple moment of $l=25$ if we correct for the beam from $I$, assuming the beam is not known to the extent to which we have considered in this paper.
\end{abstract}

Key words: methods: statistical-techniques: interferometric-diffuse radiationcosmology: observations.

\section{INTRODUCTION}

Over the last two decades, the optical spectroscopic data released from the 2-degree-Field Galaxy Redshift Survey $\left(2 \mathrm{dF}^{1}\right)$ and Sloan Digital Sky Survey $\left(\mathrm{SDSS}^{2}\right.$ ) has been used to produce 3D maps of the large-scale structure of the Universe. These optical galaxy redshift surveys are done by detecting millions of individual galaxies, determining the redshift for each and then using these to estimate their power spectrum (Santiago et al. 1995, 1996; Peterson et al. 2009).

Meanwhile, recent work in radio astronomy on $21 \mathrm{~cm}$ intensity mapping (IM) (Wyithe 2008a; Santos et al. 2015;

\footnotetext{
^E-mail: philusnarh@gmail.com

${ }^{1}$ http : //www.2dfgrs.net/

${ }^{2}$ http: //www.sdss3.org/
}

Wolz et al. 2014, 2015) suggests that the power spectrum of the full intensity field $T(f, \theta, \phi)$, defined in terms of frequency $f$ and sky position $(\theta, \phi)$, can be measured directly, without actually localizing the individual galaxies. This observational technique has the advantage of measuring the joint emission of neutral hydrogen originating from a region, including radiation from faint sources, and from the diffuse intergalactic medium (IGM), which at high redshifts cannot be detected. These intensity maps have the advantage of containing spatial information that can be used to further understand the processes of structure formation as a cosmological probe since the fluctuations are correlated with underlying dark matter density fluctuations (Bass et al. 2009, p. 366). Furthermore, the observational data from different frequencies will enable us to remove the foreground from $21 \mathrm{~cm}$ observations. This is possible since the foregrounds are smooth as a function of frequency. In addition, present and 'under construction' IM instruments such as the Green Bank Telescope (GBT) (Masui, McDonald \& Pen 2010), 
Baryon acoustic oscillations In Neutral Gas Observations (BINGO) (Battye et al. 2013), and the Canadian Hydrogen Intensity Mapping Experiment (CHIME) (Bandura et al. 2014; Newburgh et al. 2014) and the capabilities of the next generation of telescopes such as dense aperture arrays for the Square Kilometre Array (SKA) (Faulkner 2011; de Vaate \& Faulkner 2012) and the under development Hydrogen Intensity and Real-time Analysis eXperiment (HIRAX) (Newburgh et al. 2016) make prospects only more promising. IM experiments can be carried out at different redshifts in autocorrelation mode, that is single dish observations as planned by BINGO (Battye et al. 2013) or interferometric observations as planned by CHIME (Newburgh et al. 2014).

Crucially, observations using the IM technique require the accurate subtraction of the foreground continuum signal from our Galaxy as well as extragalactic sources, since the H I signal is several orders of magnitude weaker. Existing foreground-subtraction techniques rely on the smoothness of this signal as a function of frequency. In the absence of instrumental corruptions, this assumption is perfectly valid. However, real-life observations are affected by direction-dependent (DD) effects, that is variations in gain amplitude and phase over the field of view $(\mathrm{FoV})$, as well as polarization leakage. These are primarily caused by the ionosphere (in the case of phase effects) and by variations in the primary beam (PB) response of the antennas. Some of these sources of error are time-dependent: The ionosphere over the array evolves as a function of time, while the beam (of an alt-azimuthally mounted telescope) rotates with respect to the sky and is also subject to pointing offsets and deformations of the dish surface induced by the wind and gravitational load on the dishes, differential heating, etc. Problems posed by the ionosphere are especially severe at lower frequencies and with large interferometric arrays such as LOFAR (Wijnholds et al. 2010; van Haarlem et al. 2013). This work concentrates rather on the effects of the PB and in particular, DD polarization leakage. 'Polarization leakage' refers to a fraction of the signal from one orthogonal polarization mode being registered by the receptor measuring the other mode. In terms of Stokes parameters, it produces an unwanted transfer of signal between the Stokes $I$ and $Q U V$ measurements. It is a particular problem for IM observations because polarized foreground signals are generally not smooth as a function of frequency (due to Faraday rotation of the $Q U$ vector). Leakage, therefore, results in a non-smooth foreground component being introduced into Stokes $I$, one that is not amenable to traditional foregroundsubtraction techniques. Correcting for leakage is a challenge since it varies both as a function of time and as a function of frequency; hence, it is expected to limit observations with the existing as well as upcoming radio telescopes presently under construction (Bhatnagar et al. 2008a). Existing approaches for mapping out DD effects such as DD solutions (Smirnov 2011) are not directly applicable, since individual galaxies are not mapped out by this experiment. Therefore, a new approach for mapping out DD effects in a statistical sense needs to be developed. This work seeks to quantify two effects: (a) the contribution of polarization leakage to the measured $\mathrm{H}$ I power spectrum, given some more or less realistic PBs and (b) the uncertainty on the estimate of (a) introduced by unmodelled differences in the PB.

The paper is organized as follows: Section 2 defines the beam modelling techniques used in this work. Here, the OSKAR ${ }^{3}$ package is used to simulate an interferometer array of stations, each of which is an aperture array of dipoles such that this aperture array is sim-

${ }^{3}$ http: //www.oerc.ox.ac.uk/ska/oskar2/ ply a convenient model for the seven-dish Karoo Array Telescope $(\mathrm{KAT}-7)^{4}$ that can be perturbed in known ways. In addition, we validate these modelled beams with holography-measured beams of the Karl G. Jansky Very Large Array $\left(\mathrm{VLA}^{5}\right)$ in order to estimate the perturbation inaccuracies of the OSKAR beam model. Section 3 discusses briefly the components of the Galactic foregrounds and their contribution to polarization measurements. This section also continues to present a simulation of the IM experiment using convolution techniques and then presents on angular power spectrum estimation in the spherical harmonic domain. Results and analysis are outlined in Section 4. Finally, a summary of this paper is presented in Section 5.

\section{PRIMARY BEAM MODELLING}

Various techniques and software packages for modelling the primary beam response of an antenna have been developed over the years. These range from simple geometrical ray tracing implemented in cassbeam ${ }^{6}$ - a Cassegrain antenna simulator (Brisken 2003), to sophisticated EM-modelling techniques incorporated in the commercially available GRASP ${ }^{7}$ and FEKO $^{8}$ software suites. The latter two options produce the most accurate results but are quite expensive in computational and commercial terms.

Since the purpose of this work is to study the observational effects of $\mathrm{PB}$ distortion, there is the need to develop a recipe for computing both an ideal beam pattern and a set of many perturbed patterns representing deformations of the antenna. This is technically possible to do with GRASP or FEKO, but impractically expensive for our purposes (primarily because of the many perturbed patterns required). On the other hand, a physically precise model of the KAT-7 primary beam is not actually needed, since future IM observations will not be carried out by KAT-7. KAT-7 is a notional example that is adopted for the purposes of this study. What is rather needed is a relatively cheap way to compute ideal and perturbed beams, with perturbations that are representative of those seen in actual telescopes.

The OSKAR package (Dulwich et al. 2009) was developed to simulate primary beams of (and observations with) aperture arrays. It can compute the PB response of aperture array stations that are specified as a collection of dipoles. OSKAR is open source and takes advantage of graphics processing unit (GPU) acceleration to compute such patterns relatively quickly. Below, we show that OSKAR can be used to compute 'dish-like' PBs, by generating a geometric dipole distribution that mimics the aperture illumination function (AIF) of a dish. We stress that the resulting beam pattern is completely notional, and cannot be treated as a physically accurate model of the KAT-7 beam. It is, however, broadly representative of the dish beam. Furthermore, perturbations with respect to this ideal notional beam can be readily generated by perturbing the dipole distribution. The OSKAR approach gives us a practical way of generating such ideal and perturbed beams. As pointed out above, this is sufficient for the purposes of our work. Later in this section, we compare the $\mathrm{PB}$ perturbations produced by our approach with those

\footnotetext{
${ }^{4}$ http: //public.ska.ac.za/kat-7/

${ }^{5}$ http: //www.vla.nrao.edu/

${ }^{6}$ https://github.com/ratt-ru/cassbeam

${ }^{7}$ http://www.ticra.com/products/software/grasp

${ }^{8}$ https://www. feko.info/product-detail/overview-of

- feko
} 

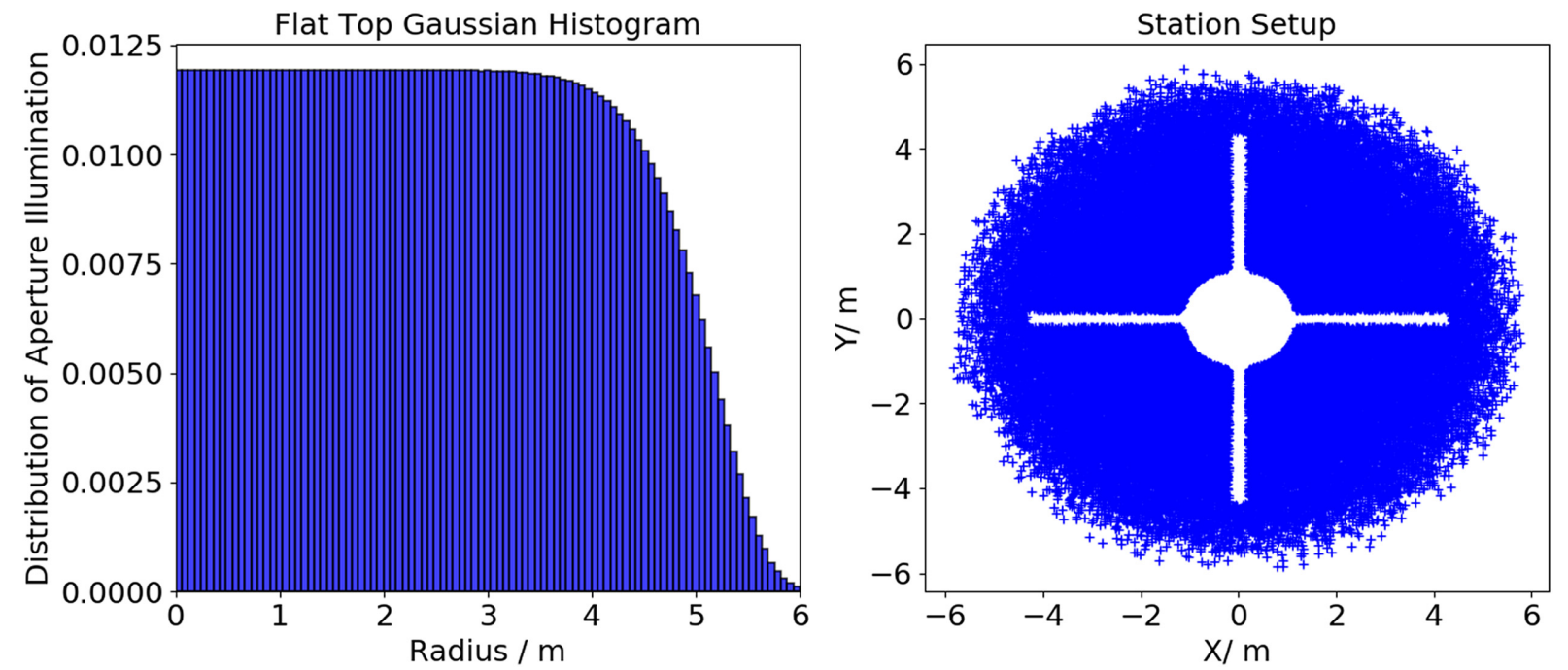

Figure 1. The aperture illumination of the dish-like surface is modelled using 80000 dipoles. Left-hand plot: The 'flat-top Gaussian' radial distribution of dipole positions, mimicking a realistic aperture illumination where the dipoles get less dense towards the edge of the dish. Right-hand plot: The resulting 2D dipole distribution with a mask applied to mimic aperture blockage.

seen in holographic measurements of VLA antennas and show that the simulated perturbations are also broadly representative.

\subsection{OSKAR beam model}

KAT-7 was produced as a forerunner to the 64-dish MeerKAT ${ }^{9}$ radio telescope array and demonstrated South Africa's ability to host the SKA (Woudt et al. 2013). The MeerKAT instrument is currently fully operational and approaching the completion of its commissioning programme (Booth \& Jonas 2012; Foley et al. 2016). When KAT-7 was in operation with only seven $12 \mathrm{~m}$ dishes scattered over a $200 \mathrm{~m}$ baseline, it was considered a compact radio telescope, in terms of both resolution and sensitivity, but now it is opposed by MeerKAT and the SKA, which occupy much larger areas. Its $L$-band radio receivers had cryogenically cooled front ends to about $70 \mathrm{~K}$ $\left(-203{ }^{\circ} \mathrm{C}\right)$ in order to increase the system's sensitivity. In addition, its configuration was superb for observing nearby galaxies, which emitted radio waves on a large scale.

In order to generate a 'dish-like' PB model using OSKAR, we aim to mimic the AIF of a KAT-7-like dish by a 2D distribution of dipole positions. There are two important features of the AIF that we need to model: a tapering off towards the edge of the dish (due to the illumination pattern of the feed) and aperture blockage by the centrally mounted feed and its four supporting struts.

To mimic illumination tapering, we generate a random distribution of 2D positions with a density that tapers off towards the edges of the dish. We do this by computing a set of positions as $x_{\mathrm{d}}=$ $R \cos (\psi)$ and $y_{\mathrm{d}}=R \sin (\psi)$, where $\psi$ is drawn from a uniform random distribution over $[0,2 \pi)$, and $R$ is drawn from a 1D radial probability distribution $f(R)$ with suitable properties. For the latter, we adopt a generalized normal (GN) distribution (Decker 1994; Pogany \& Nadarajah 2009):

$f(x)=\frac{\sqrt{s}}{2 \sigma \Gamma\left(\frac{1}{s}\right)} \exp \left(-\left|\frac{x}{\sigma \sqrt{2}}\right|^{s}\right)$

${ }^{9}$ http://www.ska.ac.za/gallery/meerkat/ where $\sigma$ is the standard deviation, $s$ is the peak factor, and $\Gamma$ is the standard gamma function defined as $\Gamma(a)=\int_{0}^{\infty} y^{a-1} \mathrm{e}^{-y} \mathrm{~d} y$. The corresponding cumulative distribution function (CDF) is given by

$$
F(x)=\left\{\begin{array}{rlc}
\frac{\Gamma\left(\frac{1}{s},\left\{\frac{x}{\sigma \sqrt{2}}\right\}^{s}\right)}{2 \Gamma\left(\frac{1}{s}\right)}, & \text { if } & x \leq 0 \\
1-\frac{\Gamma\left(\frac{1}{s},\left\{\frac{x}{\sigma \sqrt{2}}\right\}^{s}\right)}{2 \Gamma\left(\frac{1}{s}\right)}, & \text { if } & x>0
\end{array} .\right.
$$

We then draw a random number $u$ from a uniform distribution over $[0,1)$, and compute the sample $R$ as $R=\left|F^{-1}(u)\right|$. This results in a 'flat-top Gaussian' radial density distribution as shown in Fig. 1, left. The parameters of the GN distribution, $\sigma$ and $s$, control the width of the distribution and the aggressiveness of the taper. We have adopted values of $\sigma=0.82$ and $s=12.0$ to produce the radial distribution in the figure.

To mimic aperture blockage, a mask is simply applied to the $2 \mathrm{D}$ positions. This ultimately results in the dipole distribution shown in Fig. 1, right. This dipole distribution is then fed into OSKAR as the 'station layout'. For a given set of observational parameters (in particular, pointing at zenith), OSKAR then computes the station PB response. ${ }^{10}$ The resulting Jones matrix elements are shown in Fig. 2. Note how the beam pattern is broadly similar to that expected from a prime-focus dish. In particular, the first side-lobe shows the four-fold symmetry caused by the strut blockage. The presence of the phase component in Fig. 2 clearly shows that the so-called ideal beam is not that perfect since we are randomly placing the dipoles in the KAT-7 dish-like form; hence, the nominal $X$ and $Y$ dipoles are not directly orthogonal. In effect, we get the maximum rmse $\approx 0.10$ per cent perturbed inaccuracies on the dish surface as reported in Fig. 3.

\subsubsection{Jones and Mueller matrices}

The Jones (Jones 1942, 1948) formalism, originally formulated do describe optical polarization, was adapted to radio interferometry

\footnotetext{
${ }^{10}$ This takes $\approx 3$ min on a Tesla K40 GPU.
} 


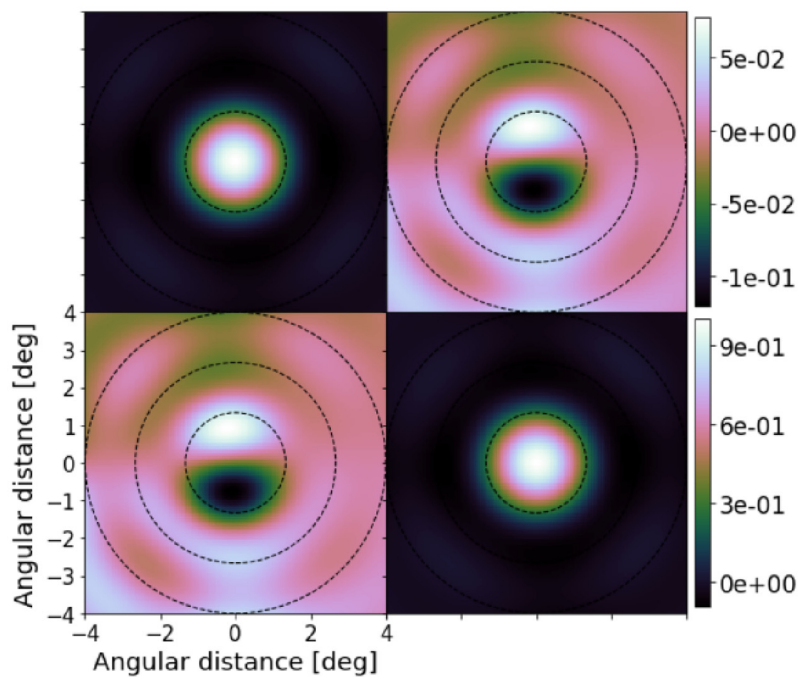

(a)

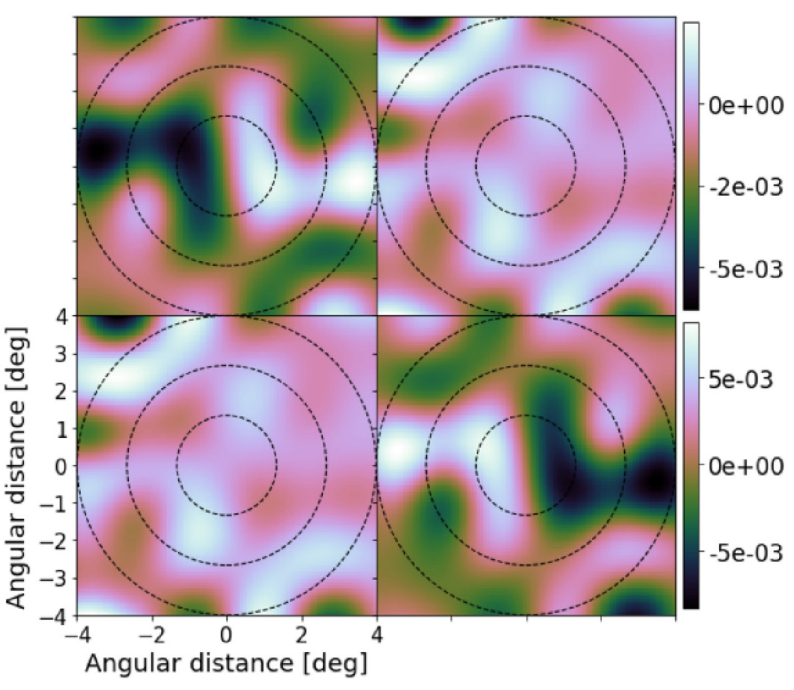

(b)

Figure 2. Jones matrix representation of the KAT-7-like beams produced by OSKAR and shown at $1 \mathrm{GHz}$ : (a) real part; (b) imaginary part. The intensity of the imaginary parts increases with fewer dipoles and becomes smaller when more dipoles are used. The four panels in (a) and (b) show $X X$ (top left), $X Y$ (top right), $Y X$ (bottom left), and $Y Y$ (bottom right). Note that the notations $X$ and $Y$ denote the horizontal and vertical linear polarised beams.
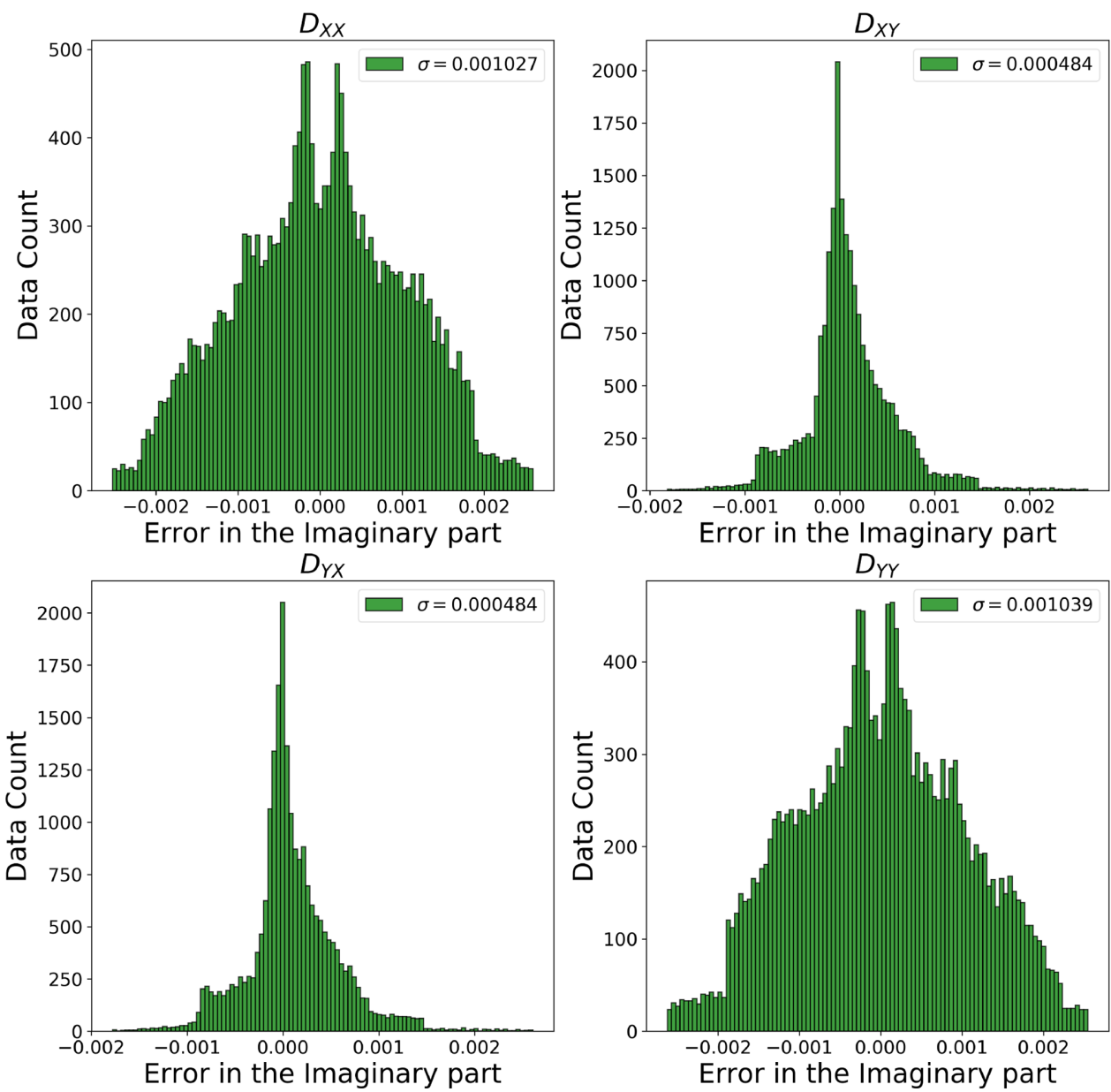

Figure 3. Histogram plots of the imaginary components in Fig. 2 showing the distribution of inaccuracies on the KAT-7 dish-like surface. 
by Hamaker, Bregman \& Sault (1996) and extended to directiondependent effects by Smirnov (2011). Here we use the derivations of the latter two works.

An electromagnetic plane wave propagating along axis $z$ can be described, at any point in space and time, by two complex amplitudes, $e_{x}$ and $e_{y}$. Conventionally, we arrange these into a column vector, $\boldsymbol{e}=\left[e_{x}, e_{y}\right]^{T}$. A single-dish observation aims to measure the pairwise coherencies of these amplitudes:

$\boldsymbol{x}=\left[\begin{array}{c}\left\langle e_{x} e_{x}^{*}\right\rangle \\ \left\langle e_{x} e_{y}^{*}\right\rangle \\ \left\langle e_{y} e_{x}^{*}\right\rangle \\ \left\langle e_{y} e_{y}^{*}\right\rangle\end{array}\right]=\left\langle\boldsymbol{e} \otimes \boldsymbol{e}^{*}\right\rangle$

where $\langle\cdot\rangle$ represents the average over a time/frequency interval, and $\otimes$ is the outer (or Kronecker) product operator. From these measured coherencies, the Stokes parameters $I Q U V$ (written as a column vector $s$ ) may be derived, by definition, as (Born \& Wolf 1980):

$\boldsymbol{s}=\left[\begin{array}{c}I \\ Q \\ U \\ V\end{array}\right]=\left[\begin{array}{c}\left\langle e_{x} e_{x}^{*}\right\rangle+\left\langle e_{y} e_{y}^{*}\right\rangle \\ \left\langle e_{x} e_{x}^{*}\right\rangle-\left\langle e_{y} e_{y}^{*}\right\rangle \\ \left\langle e_{x} e_{y}^{*}\right\rangle+\left\langle e_{y} e_{x}^{*}\right\rangle \\ -l\left(\left\langle e_{x} e_{y}^{*}\right\rangle-\left\langle e_{y} e_{x}^{*}\right\rangle\right)\end{array}\right]$.

We can rewrite this in terms of a $4 \times 4$ conversion matrix $\mathbf{S}^{-1}$ as ${ }^{11}$

$s=\left[\begin{array}{rrrr}1 & 0 & 0 & 1 \\ 1 & 0 & 0 & -1 \\ 0 & 1 & 1 & 0 \\ 0 & -l & l & 0\end{array}\right] \boldsymbol{x}=\mathbf{S}^{-1} \boldsymbol{x}$.

What the instrument actually measures is a set of pairwise correlations between two voltages induced by the EM field on two orthogonal mode feeds, $v_{a}$ and $v_{b}$. The Jones formalism assumes that these are linearly related to the EM field (i.e. that all signal propagation effects are linear). This can be written as $\boldsymbol{v}=\mathbf{J} \boldsymbol{e}$, where $\boldsymbol{v}$ is a column vector of the two voltages, and the $2 \times 2$ Jones matrix $\mathbf{J}$ describes signal propagation. The measured coherency $\boldsymbol{x}^{\prime}$ can then be written as

$\boldsymbol{x}^{\prime}=\left\langle\boldsymbol{v} \otimes \boldsymbol{v}^{*}\right\rangle=\left(\mathbf{J} \otimes \mathbf{J}^{*}\right)\left\langle\boldsymbol{e} \otimes \boldsymbol{e}^{*}\right\rangle=\left(\mathbf{J} \otimes \mathbf{J}^{*}\right) \boldsymbol{x}$,

and the measured Stokes parameter vector $\boldsymbol{s}^{\prime}$ relates to the original Stokes vector via the so-called Mueller matrix $\mathrm{M}$ :

$s^{\prime}=\mathbf{M} s=\mathbf{S}^{-1}\left(\mathrm{~J} \otimes \mathrm{J}^{*}\right) \mathrm{S} s$.

For the purposes of this work, we ignore all propagation effects except the primary beam. In the context of this paper, the Mueller matrix refers to the Mueller matrix of the primary beam. This matrix is direction dependent (i.e. each direction of arrival will have its own matrix associated with it). The total Stokes flux measured by a single dish observation is then an integration over the FoV:

$\boldsymbol{s}_{\text {tot }}^{\prime}=\iint_{l m} \mathbf{M}(l, m) \boldsymbol{s}(l, m) \mathrm{d} l \mathrm{~d} m$,

\footnotetext{
${ }^{11}$ This follows Smirnov (2011) in defining $\mathbf{S}$ as the conversion matrix between Stokes vectors and coherency vectors, $\boldsymbol{v}=\mathrm{S} s$. Conversely, $\mathbf{S}^{-1}$ operates in the opposite direction. Note that Hamaker et al. (1996) use T to refer to $\mathbf{S}^{-1}$.
}

where the integration is, in principle, over the entire celestial sphere, but in practice, since the Mueller matrix becomes negligibly small outside of a certain FoV, it can be replaced by a 2D integral over the tangent plane $l m$.

The Mueller matrix $\mathbf{M}(l, m)$ corresponding to our KAT-7-like dish (Fig. 4a) can be derived from the Jones matrix $\mathbf{J}(l, m)$ of Fig. 2. Note the physical meaning of the matrix elements. The ondiagonal terms of the Jones matrix describe the sensitivity of each feed, as a function of direction, to its matched EM field component. The off-diagonal terms describe leakage, i.e. the sensitivity of the feed to the nominally orthogonal EM field component. This leakage is due to mechanical and electronic imperfections in the antennas and feeds. The diagonal terms of the Mueller matrix describe the sensitivity of the measured Stokes $I Q U V$ components to their true counterparts, as a function of direction. The off-diagonal terms describe spurious leakage between the measured Stokes components. We can schematically write this as

$\mathbf{M}=\left[\begin{array}{cccc}I \rightarrow I^{\prime} & Q \rightarrow I^{\prime} & U \rightarrow I^{\prime} & V \rightarrow I^{\prime} \\ I \rightarrow Q^{\prime} & Q \rightarrow Q^{\prime} & U \rightarrow Q^{\prime} & V \rightarrow Q^{\prime} \\ I \rightarrow U^{\prime} & Q \rightarrow U^{\prime} & U \rightarrow U^{\prime} & V \rightarrow U^{\prime} \\ I \rightarrow V^{\prime} & Q \rightarrow V^{\prime} & U \rightarrow V^{\prime} & V \rightarrow V^{\prime}\end{array}\right]$.

Fig. 4(a) displays the KAT-7-like modelled beams produced from OSKAR in Mueller matrix form. For example, the elements $I$ $\rightarrow Q, U, V$ in the $4 \times 4$ images describe how much of the Stokes $I$ intensity leaks into the polarization components $Q, U$, $V$ while $Q \leftrightarrow U, Q \leftrightarrow V, U \leftrightarrow V$ are the cross-polarization leakage terms.

These modelled beams are then corrupted with two kinds of errors. The first type was to introduce systematic and time-variable gain and phase errors. The nominal purpose of this in OSKAR is to simulate per-element gain and phase error before the beam former so that the beam-forming weight $B^{\mathrm{w}}$, for a particular beam direction $\left(\theta_{\mathrm{bm}}, \phi_{\mathrm{bm}}\right)$, with dipole position $(x, y, z)$ and time $t$ becomes

$B^{\mathrm{w}}(u)=B_{\mathrm{geo}}^{\mathrm{w}}(u)\left(G_{0}+G_{\text {error }}\right) \exp \left(j\left[\phi_{0}+\phi_{\text {error }}\right]\right)$

where $u=\left(\theta_{\mathrm{bm}}, \phi_{\mathrm{bm}}, x, y, z, t\right), G_{\text {error }}$ and $\phi_{\text {error }}$ are pseudo-random values at each time-step $t$ using a Gaussian distribution with standard deviations $G_{\text {std }}$ and $\phi_{\text {std }}$ respectively. The complex multiplicative factor applied to each element is denoted by the parameters gain $G_{0}$ and phase $\phi_{0}$, respectively. This complex factor joins with the geometric beam-forming weight $B_{\text {geo }}^{\mathrm{w}}$ to produce the array factor to evaluate the station beam at each source position. For the purpose of our 'disk-like' simulation, we introduced $5^{\circ}$ phase error and 10 per cent gain error into the beam-forming weight to distort the beams as shown in Fig. A1(a). These sorts of errors represent imperfections in the parabolic reflector surface (which, in real life, result in amplitude and phase errors over the aperture). The second error introduced was to uniformly change the orientation of the dipoles to create systematic error feed angle displacement as presented in Fig. A1(b). Figs 4(b) and (c) show the beam errors produced by computing the differences between the true modelled beams in Fig. 4(a) and the two distorted beams in Figs A1(a) and (b), respectively. The on-diagonal components of these beam errors represent the residual leakages and the off-diagonals show the residual systematic leakages. The maximum residual leakages produced in Figs 4(b) and (c) are $\simeq 20$ per cent and 10 per cent, respectively.

In order to see whether the level of beam distortion introduced in this manner is realistic, we compare the beam patterns obtained via 


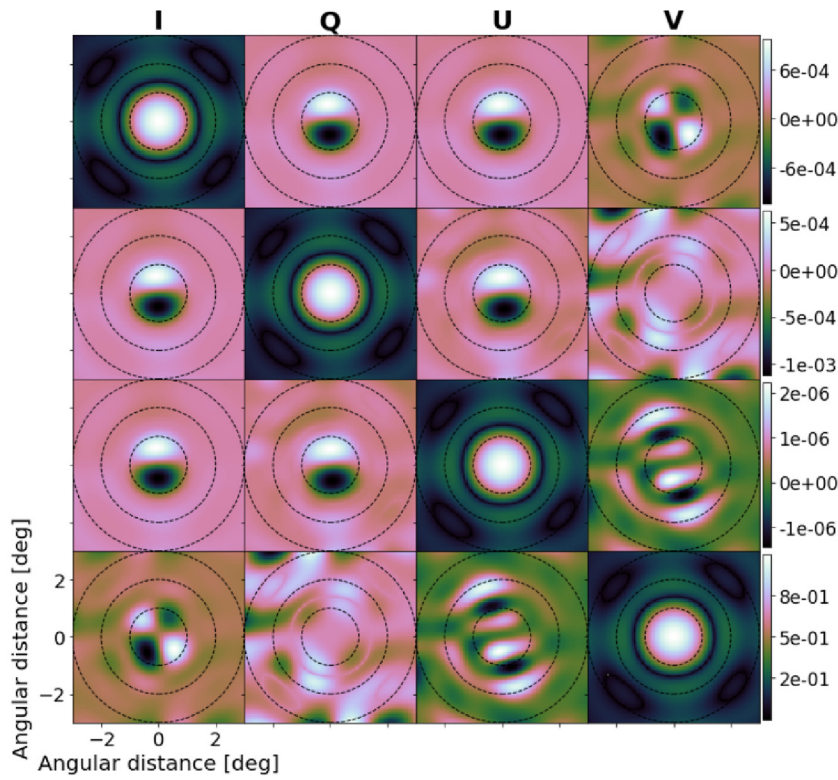

(a)

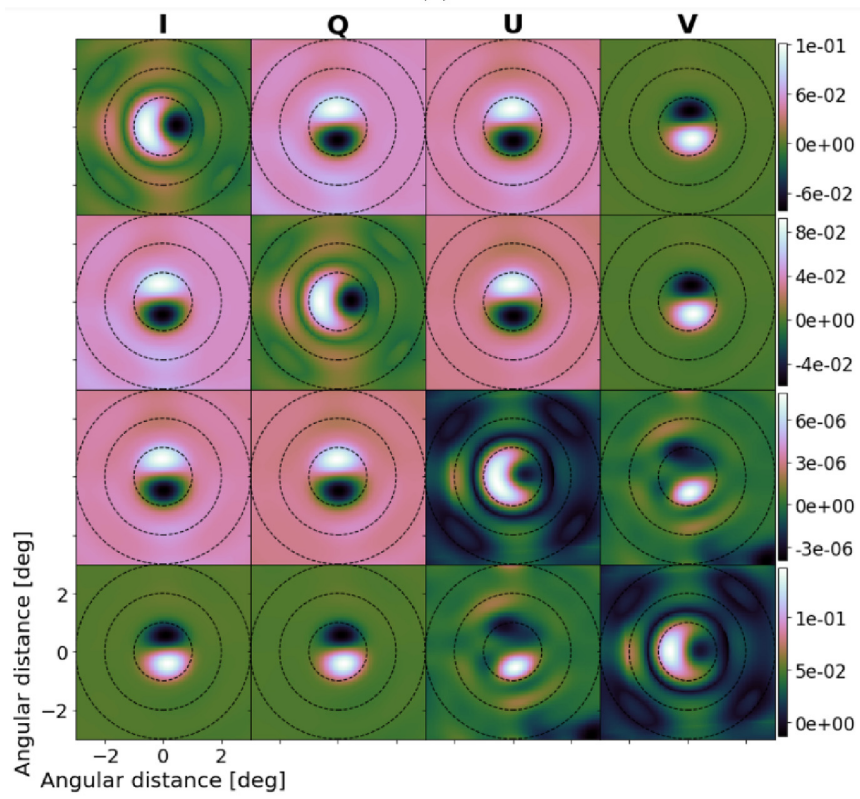

(c)

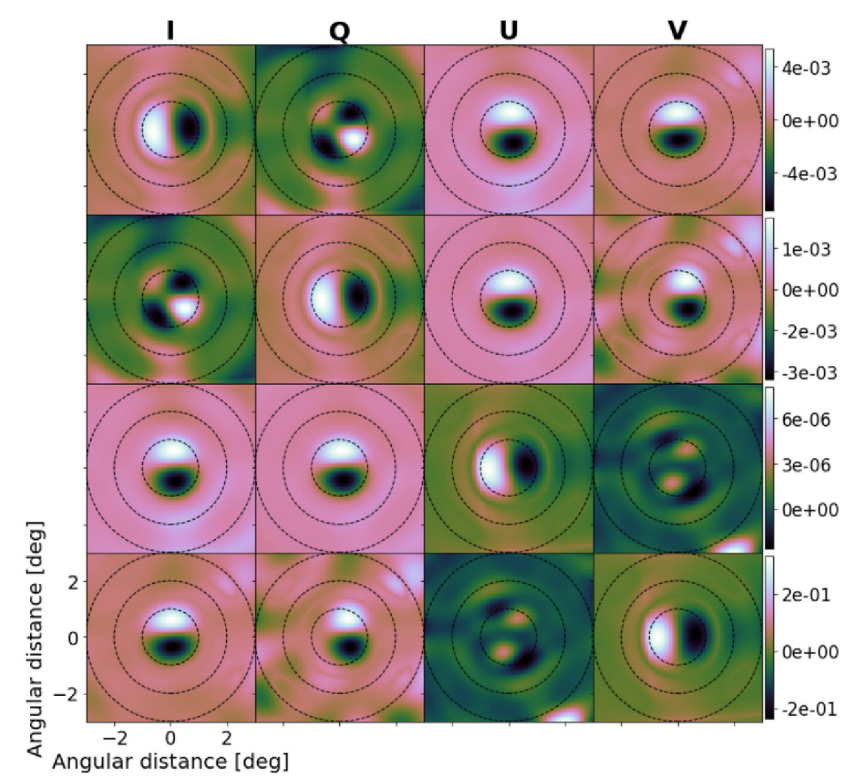

(b)

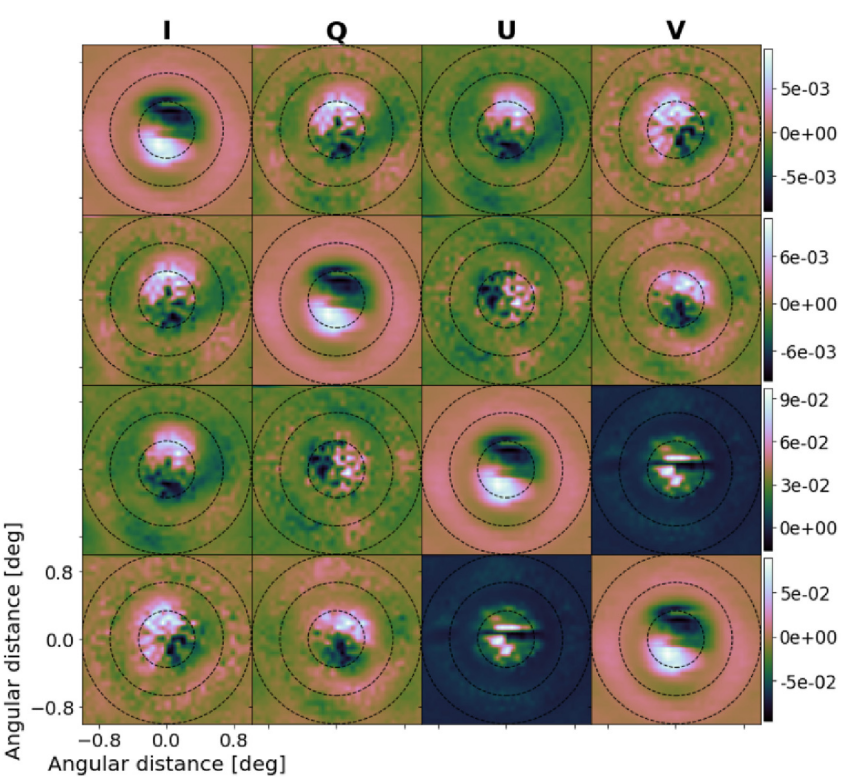

(d)

Figure 4. Mueller matrix representations of full polarization beams produced at $1 \mathrm{GHz}$. (a) $4 \times 4$ images of KAT-7 uncorrupted OSKAR beams. (b) Fractional differences between the uncorrupted OSKAR beams in Fig. 4(a) and the gain and phase error beams in Fig. A1(a). (c) Fractional differences between uncorrupted OSKAR beams in Fig. 4(a) and the dipole orientation error beams in Fig. A1(b). (d) Fractional differences between VLA holography-measured beams in Figs A2(a) and (b).

holography measurements of two different VLA dishes as presented in Figs A2(a) and (b). The measurement technique employed in producing these beams is based on the EVLA Memo (Perley et al. 2015), which consists of the utilization of the Fourier transform relation between the complex far-field (i.e. amplitude and phase) radiation pattern of an antenna $\chi(u, v)$ and the complex aperture distribution $\zeta(l, m)$ :

$\chi(u, v)=\iint_{-\infty}^{+\infty} \zeta(l, m) \exp \{i 2 \pi(l u+m v)\} \mathrm{d} l \mathrm{~d} m$ where $u=x / \lambda$ and $v=x / \lambda$ denote the rectilinear coordinates in wavelength, in the aperture plane. The coordinates $(l, m)$ are the direction cosine with respect to the aperture plane. The difference between the two holography measurements in Fig. 4(d) corresponds to the real-life differences between the PB patterns of two nominally identical dishes. Note how these beam errors give a maximum residual leakage of $\simeq 10$ per cent. This demonstrates that our beam distortion procedure, using the error values we have adopted, results in physically realistic PB distortion levels.

Having thus armed ourselves with an instrumental model, we now proceed to discuss the nature of our simulated sky. 


\section{SIMULATION}

The synchrotron emission from the Galaxy dominates at low microwave frequencies $(\lesssim 30 \mathrm{GHz})$, whilst that of thermal dust emission is at higher frequencies $(\lesssim 70 \mathrm{GHz})$. Between these two components in frequency lies the thermal free-free and non-thermal dust emissions, which are formed as a result of spinning dust grains (Planck Collaboration VIII 2016b). Detailed discussion of the components of the Galactic foregrounds, paying particular attention to their contributions to the polarization measurements, can be found in Alonso et al. (2015), Kiyotomo (2014), Jelić et al. (2010), Santos et al. (2015), and Wolz et al. (2014).

Future $21 \mathrm{~cm}$ experiments will operate in a window that is sampled sparsely by current observations. In particular, we are interested in the properties of the unpolarized and polarized foregrounds at small frequency separations, but existing full-sky observations around $1 \mathrm{GHz}$ are generally low resolution, unpolarized, and separated by large frequency intervals. For simulations to be a useful tool, they need to produce sufficiently challenging foregrounds, not only reproducing the properties we see in observations, but also incorporating unobserved structure of small angular and frequency scales, as well as the spectral variations of the polarized sky caused by emission at varying Faraday depth through the Galaxy.

Low-frequency all-sky polarization data is limited, though upcoming surveys like GMIMS (Wolleben et al. 2009) will change this. The most useful full-sky polarization measurements come from 1.4 GHz surveys (Wolleben et al. 2006; Testori, Reich \& Reich 2008) and the WMAP $23 \mathrm{GHz}$ (Bennett et al. 2013) and Planck $30 \mathrm{GHz}$ maps (Planck Collaboration VIII 2016a). However, because of the uncertain amount of instrumental depolarization in the former and the much higher frequencies of the latter, these are more useful to tell us about the general properties of the polarized sky (such as the high galactic latitude polarization fraction; see Kogut et al. 2007) and less about the specific realization on the sky. With these limitations in mind, we aim not to reproduce existing polarisation, but to include the relevant difficulties for $21 \mathrm{~cm}$ intensity mapping.

To generate the foreground simulations used in this paper (shown in Fig. 5), we use the CORA package ${ }^{12}$, which is described in Shaw et al. $(2014,2015)$. In brief, the procedure used to produce the simulations is as follows:

(i) A 3D base map for the unpolarized sky is produced by extrapolating the $408 \mathrm{MHz}$ Haslam et al. (1982) map to the requested set of frequencies using the spectral index of the map of MivilleDeschênes et al. (2008a), which was produced by comparing the Haslam map with WMAP $23 \mathrm{GHz}$ polarization data, assuming that both are dominated by synchrotron emission. This produces a map with power-law spectral behaviour with angular fluctuations on scales $\gtrsim 1^{\circ}$.

(ii) Angular and spectral fluctuations are added into the 3D maps according to an angular power spectrum.

$C_{\ell}\left(v, v^{\prime}\right) \propto\left(\frac{\ell}{\ell_{0}}\right)^{-2.8}\left(\frac{v v^{\prime}}{v_{0}^{2}}\right)^{-2.8} \exp \left[-\frac{1}{2}\left(\frac{\ln v / v^{\prime}}{4}\right)^{2}\right]$,

taken from Santos, Cooray \& Knox (2005). We use positiondependent scaling on large scales to ensure the fluctuations match their observed variance across the sky (La Porta et al. 2008), and ensure that we don't add in additional fluctuations on scales con- strained by the Haslam map by projecting out the the dominant eigenmodes on these scales.

(iii) To simulate the polarized sky, we use the ideas of Faraday rotation measure synthesis (Brentjens \& de Bruyn 2005) and a simple model of the distribution of emission in Faraday depth across the full sky, which we then integrate over to generate the polarized output at each desired frequency. To produce the emission in Faraday space, we use the rotation measure map of Oppermann et al. (2012) to indicate the characteristic scale of the distribution of emission in Faraday depth. We tune the amplitude and correlation properties in Faraday space to crudely reproduce the polarization fraction in the WMAP $23 \mathrm{GHz}$ map and the $1.4 \mathrm{GHz}$ surveys. The polarization directions are generated as a Gaussian random field at each Faraday depth. For the interested reader, more details of this are found in Shaw et al. (2015).

(iv) Known bright point sources on the sky are included explicitly, with their polarizations Faraday rotated to the desired frequencies using the Oppermann et al. (2012) map. Faint sources $(S<10 \mathrm{Jy}$ at $151 \mathrm{MHz}$ ) are randomly generated.

The smoothed foreground maps in Fig. 5 are represented by a sample of Hierarchical Equal Area isoLatitude Pixelation $\left(\right.$ HEALPix $^{13}$ ) (Górski et al. 2005a) of the sphere at a resolution of $N_{\text {side }}=512$. Other techniques for simulating full-sky radio emission are presented by de Oliveira-Costa et al. (2008a), Geil, Gaensler \& Wyithe (2011), Jelić et al. (2008), the Polarbear Collaboration: P. A. R. Ade et al. (2014), Tucci et al. (2002). This paper also considered the synchrotron emissions to be intrinsically linearly polarized and therefore, ignored the Stokes $V$ part in Fig. 5 and all other $V$ terms in the full polarization beams discussed in Section 2.

\subsection{Full-sky convolution}

To perform an IM experiment, the radio telescope(s) is pointed at different patches of the sky so that the instrument can measure the overall intensity emerging from patches from the autocorrelation of the radio signal, as a function of frequency. In order to emulate this observation technique in our IM simulation, the discrete convolution in equation (12) is used to measure the intensities of the full-sky synchrotron maps in Fig. 5. Let $(\theta, \phi)$ denote the celestial coordinates of the foregrounds of the sky such that $\boldsymbol{B}$ are the fully polarized beams and $\boldsymbol{f}_{\text {sky }}$ are the foregrounds of the sky. We can then model the convolved foregrounds to be

$$
\begin{aligned}
\boldsymbol{F}^{\text {conv }}(\theta, \phi) & =\boldsymbol{B}(\theta, \phi) \otimes \boldsymbol{f}_{\text {sky }}(\theta, \phi) \\
& =\sum_{\left(\theta^{\prime}, \phi^{\prime}\right)=\lfloor(\theta, \phi)\rceil} \boldsymbol{B}\left(\theta^{\prime}-\theta, \phi^{\prime}-\phi\right) . \boldsymbol{f}_{\text {sky }}\left(\theta^{\prime}, \phi^{\prime}\right)
\end{aligned}
$$

where $\left(\theta^{\prime}, \phi^{\prime}\right) \leq$ npix and the symbol \lfloor\rceil denotes the nearest pixels. The measured foreground pixel values $\boldsymbol{F}^{\text {conv }}(\theta, \phi)$ of the discrete function $\boldsymbol{F}^{\text {conv }}$ for any particular $(\theta, \phi)$ follows by multiplying each foreground pixel value $f_{\text {sky }}(\theta, \phi)$ of the discrete function $f_{\text {sky }}$ with a beam $\boldsymbol{B}\left(\theta^{\prime}-\theta, \phi^{\prime}-\phi\right)$ between a particular $\left(\theta^{\prime}, \phi^{\prime}\right)$ and varying $(\theta, \phi)$. Thus, each pixel value $\boldsymbol{F}^{\mathbf{c o n v}}(\theta, \phi)$ of the function $\boldsymbol{F}^{\text {conv }}$ is a weighted mean of the pixel values $\boldsymbol{f}_{\text {sky }}(\theta, \phi)$ with weights $\boldsymbol{B}\left(\theta^{\prime}-\theta, \phi^{\prime}-\phi\right)$ defined by the function $\boldsymbol{B}$. If we take the modelled beams $\boldsymbol{B}$ in Fig. 4(a) to convolve the full-sky polarization maps $\left(f_{\text {sky }}\right)$ in Fig. 5, we obtain the convolved maps $\boldsymbol{F}^{\text {conv }}$ in Fig. 6. The same approach is repeated using the distorted beams in Figs A1(a) and (b) and also, the holography-measured beams in Figs A2(a) and 

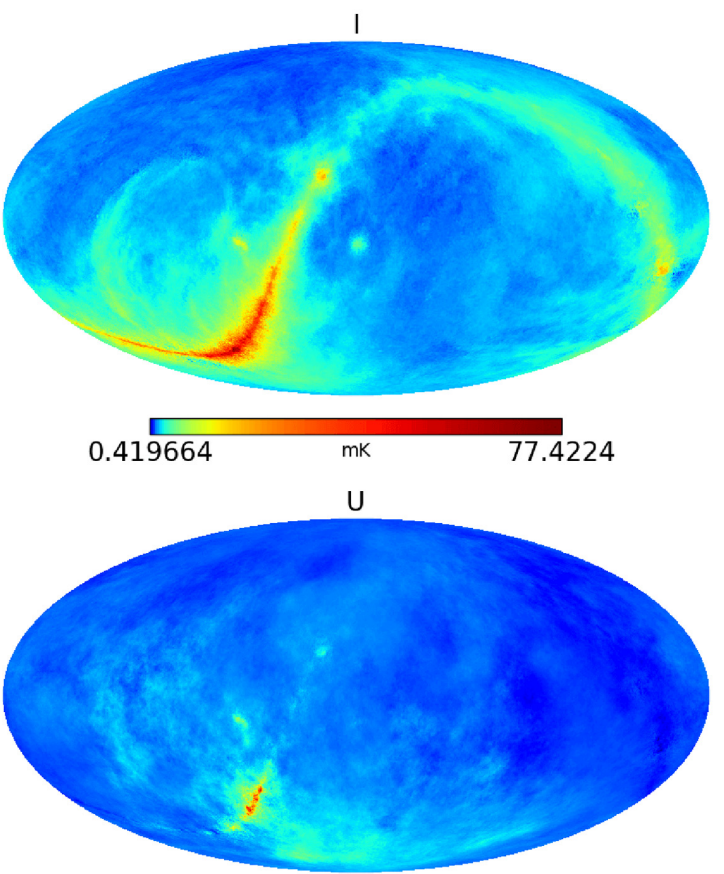

$-2.03096$

6.81405
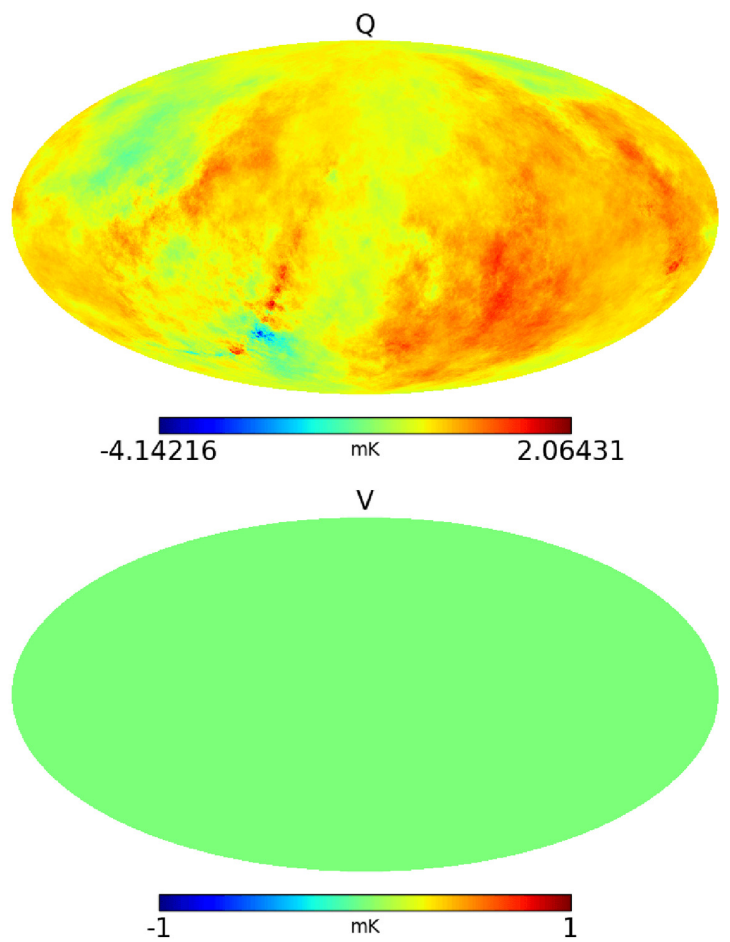

Figure 5. $1000 \mathrm{MHz}$ full-sky synchrotron maps simulated by using m-mode formalism. These synchrotron maps characterize the full-sky polarization maps for our low-resolution simulated observations and are presented here in the Mollweide projection form defined by equatorial coordinates in terms of Stokes parameters $I, Q, U$, and $V$.
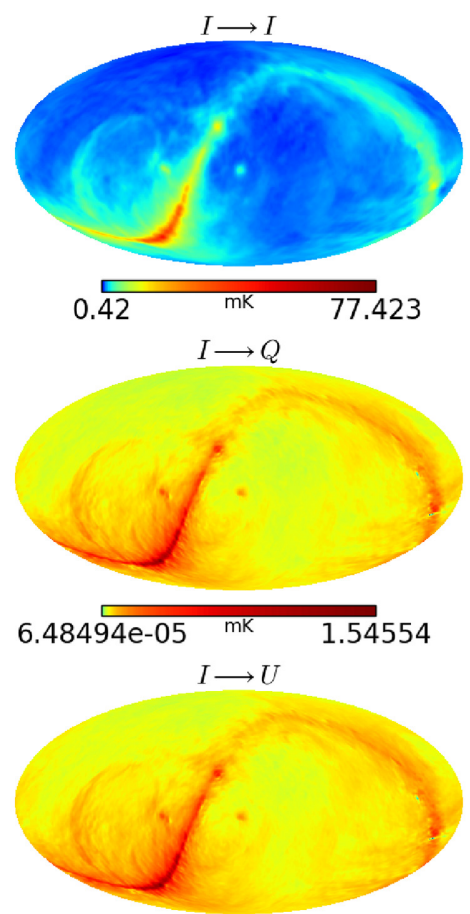

$8.74344 \mathrm{e}-05 \quad \mathrm{mK} \quad 3.09361$
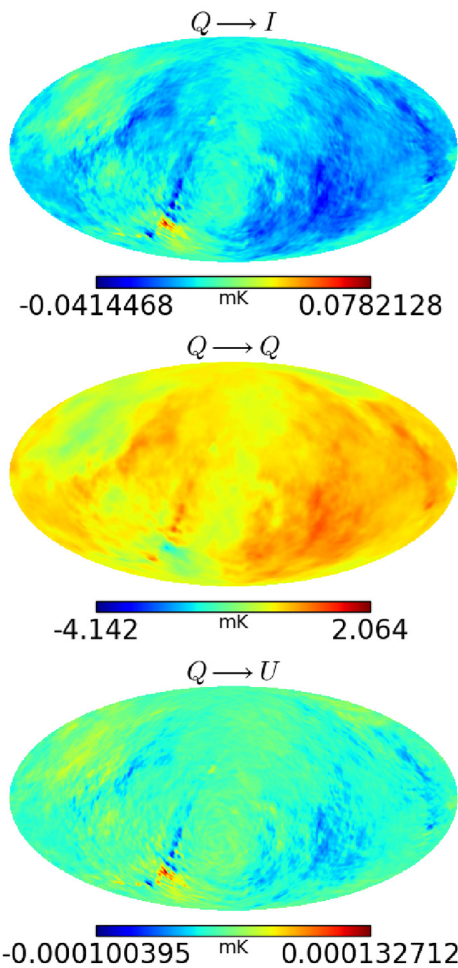
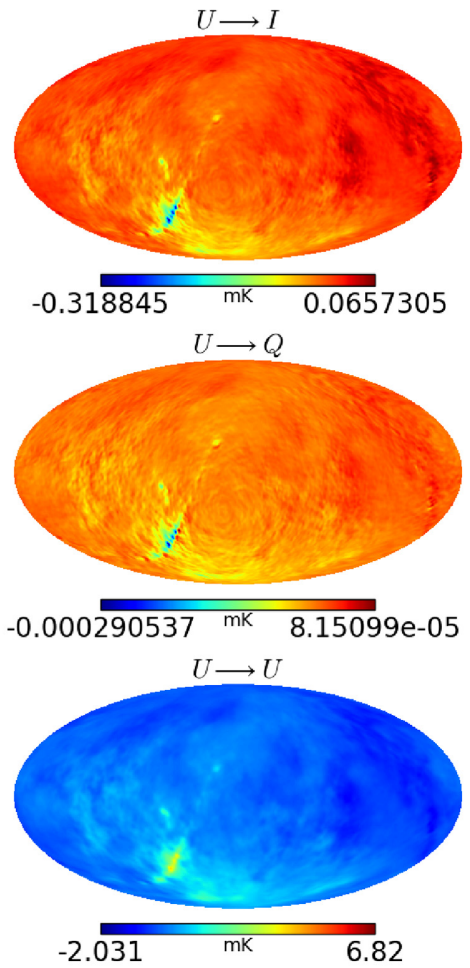

Figure 6. Convolved full-sky polarization maps using the non-distorted OSKAR beams. For example, we used the $m_{\text {II }}$ beam in Fig. 4(a) to convolve Stokes $I$ in Fig. 5 and produce the convolved map $I \rightarrow I$. Then we used the $m_{Q I}$ beam to convolve Stokes $Q$ to obtain the convolved map $Q \rightarrow I$. Also, using the $m_{U I}$ beam to convolve Stokes $U$ we produced the convolved map $U \rightarrow I$. The other convolved maps are produced in the same manner using their respective beams. 
(b) to produce their respective convolved maps. Note how the original spatial distributions of the foregrounds in Fig. 5 are maintained in the diagonals of the convolved maps displayed in Fig. 6. These happen when we simulate the full-sky maps with the gain terms in the diagonals of our beams.

In IM experiments, what we are actually interested in is measuring the total intensity of a signal. Therefore, in Section 3.2, we present a mathematical model of the convolved power spectrum using the angular power spectrum approach to describe the spatial distribution of the measured foregrounds in a spherical harmonic domain.

\subsection{Angular power spectrum}

In CMB studies (White 1998; Saha, Jain \& Souradeep 2006; Souradeep, Saha \& Jain 2006; Wolz et al. 2015; Abitbol, Hill \& Johnson 2016; Krachmalnicoff et al. 2016), it is a common practice to characterize the distribution of flux in a sphere with the angular power spectrum. The same approach is employed in this paper to describe the diffuse foreground intensity over spherical harmonics $Y_{l, m}$.

Consider the foreground of the sky is emitted by our own Galaxy or the distribution galaxies emitting $21 \mathrm{~cm}$ with an intensity equivalent to $T(\hat{\sigma})$. We can measure the total source emission temperature, $T(\hat{\sigma})$, in each sky pixel and represent the distribution as an expansion in 2D spherical harmonics:

$T(\hat{\boldsymbol{\sigma}})=\sum_{l=0}^{\infty} \sum_{m=-l}^{l} a_{l m} Y_{l m}(\hat{\boldsymbol{\sigma}})$

where $\hat{\sigma} \equiv(\psi, \xi)$ is the unit vector in some direction in the sky and $Y_{l m}(\hat{\boldsymbol{\sigma}})$ are the spherical harmonic functions evaluated in the direction $\hat{\sigma}$ such that they form a complete orthonormal set on the unit sphere and can be expressed as

$Y_{l m}(\psi, \xi)=(-1)^{m} \sqrt{\frac{2 l+1}{4 \pi} \frac{(l-m) !}{(l+m) !}} P_{l}^{m}(\cos \psi) e^{i m \xi}$

In equation (14), the indices $l=0, \ldots, \infty$ and $-l<m<l$ with $P_{l}^{m}$ denoting the Legendre polynomials. $l$ is known as the 'multipole', which denotes a given angular scale $\gamma$ in the sky, where $\gamma \simeq 180^{\circ} /$. The coefficients $a_{l m}$ in equation (13)

$a_{l m}=\int_{\psi=-\pi / 2}^{\pi / 2} \int_{\xi=0}^{2 \pi} T_{l m}(\hat{\boldsymbol{\sigma}}) Y_{l m}^{*}(\hat{\boldsymbol{\sigma}}) \mathrm{d} \xi \mathrm{d} \psi$

are related to what we normally do in the Fourier space.

Consider any two pixels; then the correlation function of the temperatures is expressed as;

$C_{c r}(\Theta)=\left\langle T\left(\hat{\sigma}_{i}\right) T\left(\hat{\sigma}_{j}\right)\right\rangle, \quad \Theta=\sigma_{i} . \sigma_{j}$

where the brackets \langle\rangle denote averaging over $2 l+1$ values of $m$. Equation (16) strictly relies on the separation angle between two sources as discussed in Schramm \& Galeotti (1997, p. 78) and therefore can be rewritten in terms of Legendre polynomials:

$C_{\mathrm{cr}}(\Theta)=\sum_{l=0} \frac{2 l+1}{4 \pi} C_{l} P_{l}(\cos \Theta)$.

From equation (17), we can estimate the statistical distribution of the angular power spectrum $\hat{C}_{l}$ of the entire sky in terms of $a_{l m}$ :

$\hat{C}_{l}=\frac{1}{2 l+1} \sum_{m}\left|\hat{a}_{l m}\right|^{2}, \quad-l<m<l$.
In this paper, we used anafast in the HEALPix library to compute the autopower spectrum $\hat{C}_{l}$ of foregrounds of the sky in Section 3.1 by executing an approximate discrete point-set quadrature on a sphere sampled at the HEALPix pixel centres. Spherical harmonic transforms are then computed using recurrence relations for Legendre polynomials on co-latitude $\psi$ and fast Fourier transforms on longitude $\xi$.

\section{RESULTS AND ANALYSIS}

The measured full-sky maps of $\left\{I^{\mathrm{T}}, Q^{\mathrm{T}}, U^{\mathrm{T}}\right\}$ (reported in row 1), $\left\{I^{\mathrm{GP}}, Q^{\mathrm{GP}}, U^{\mathrm{GP}}\right\}$ (reported in row 2), and $\left\{I^{\mathrm{XY}}, Q^{\mathrm{XY}}, U^{\mathrm{XY}}\right\}$ (reported in row 3 ) in Fig. 7 are generated by convolving both the true (in Fig. 4) and perturbed model beams (in Figs A1 a and b, respectively) with the foregrounds and then adding up the respective maps in each row of the convolved Stokes terms. Note the similarities between these measured maps: If we compute the differences between the maps in the first and second rows and also, the first and third rows, we obtain the corresponding error maps in rows 4 and 5, respectively. Obviously, these simulated maps in Fig. 7 are not the same and this is even confirmed by the systematic differences presented in Fig. 8, between the true convolved maps in Fig. 6 and the corrupted maps due to errors introduced in the gain and phase of the PBs. We then repeat the same approach using the VLA measured beams displayed in Fig. A2 to obtain the systematic error terms in Fig. B1 and the overall measured full-sky maps reported in Fig. B2. In this paper, the idea to use the VLA holography beams to also convolve the full-sky polarization maps is to compare the error we make in the power spectrum estimation if a model beam is considered, whilst the foreground is actually convolved with a 'real' beam.

The autopower spectra presented in Fig. 9 estimate the density of the measured foregrounds at different multipole moments. Note how the beam power in each plot of both OSKAR and the holographicmeasured beams is normalized to 1 . It is computed by finding the quotient of the power spectrum of the convolved sky map and the original sky map. In addition, note also in all cases the PB effect of the convolved power spectrum. The OSKAR beam power plots in Stokes $I, Q$, and $U$ converge at a multipole moment of $l=60$. This value relates to an angular scale of $3.0^{\circ}$ on the sky whilst the power spectra of the VLA beams converge just at a multipole moment of $l=90$, giving an angular scale of $2.0^{\circ}$ on the sky. The change in the value of multipole moments is because of different dish sizes, which also results in producing different beamwidths. Furthermore, the angular scales computed are equivalent to the beam sizes used to convolve the original maps in Fig. 5. Note, even though we used two different aperture sizes for the simulation, the effect of these two PBs on the convolved power spectra of Stokes $I, Q$, and $U$ remains unaltered. This shows that in IM experiment, where we measure the collective emission from many sources, smaller and relatively cheaper instruments can be used. In this study, the measured values for the convolved power spectra of Stokes $I, Q$, and $U$ in both cases are $10,0.1$, and $0.1 \mathrm{mK}^{2}$, respectively. Observe carefully how these values in Fig. 9 actually predicted the foreground's temperature of the true sky. Hence, the power spectrum of the corresponding errors in $I, Q$, and $U$ due to perturbation of the beams are $\approx 0.01,10^{-4}$, and $10^{-5} \mathrm{mK}^{2}$, respectively.

In IM experiments, the $\mathrm{H}$ I signal is measured in Stokes $I$, so we are particularly interested in the total intensity and the leakages from polarization into Stokes $I$ (i.e. $|Q+i U| \longrightarrow I$ ). Fig. 10 shows how $|Q+i U| \longrightarrow I$ and the error in the Stokes $I$ map affect the $\mathrm{H} \mathrm{I}$ signal. Here, a spherical power spectrum of the simulated model of 


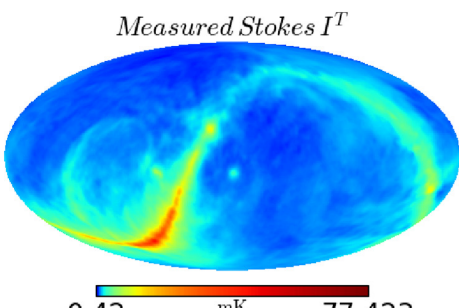

$0.42 \quad \mathrm{mK} \quad 77.423$
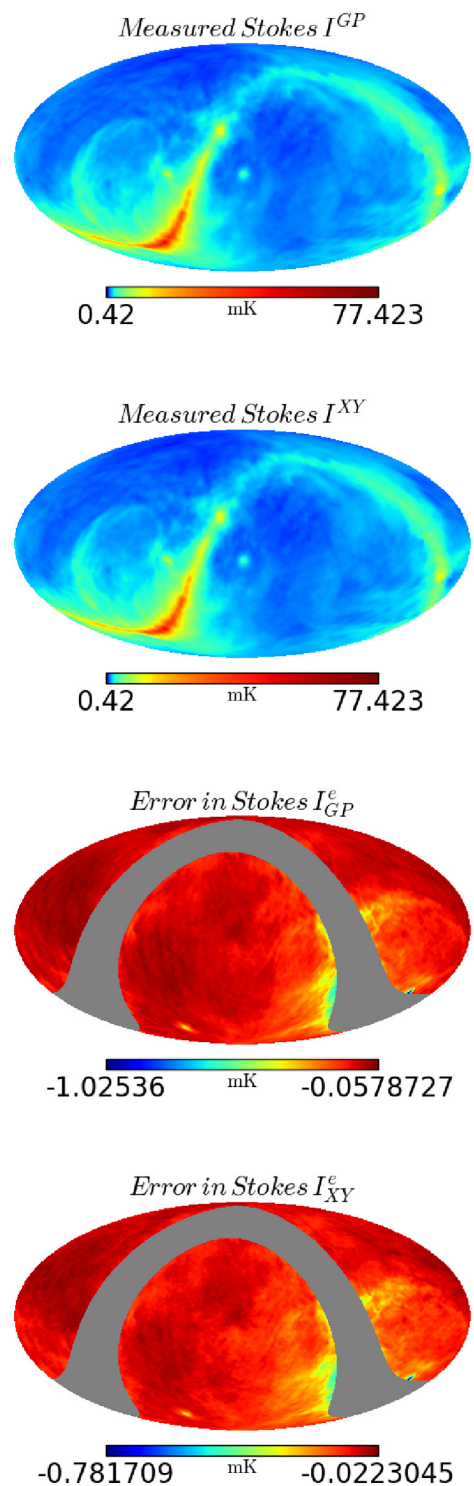

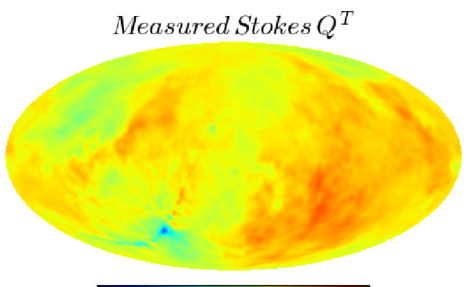

$-4.142 \quad \mathrm{mK} \quad 2.064$
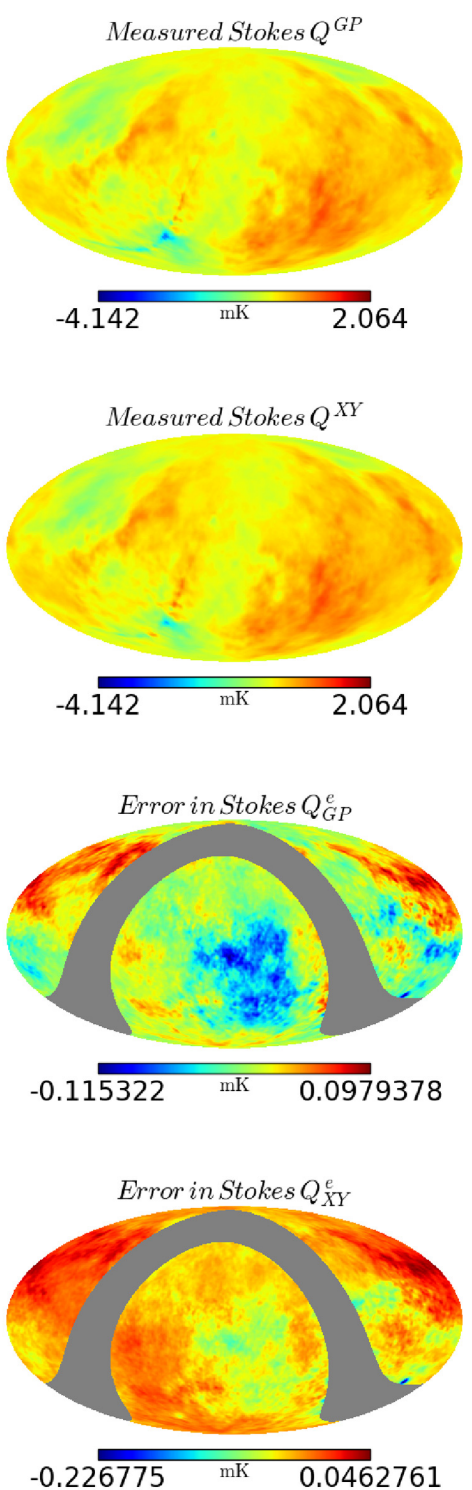
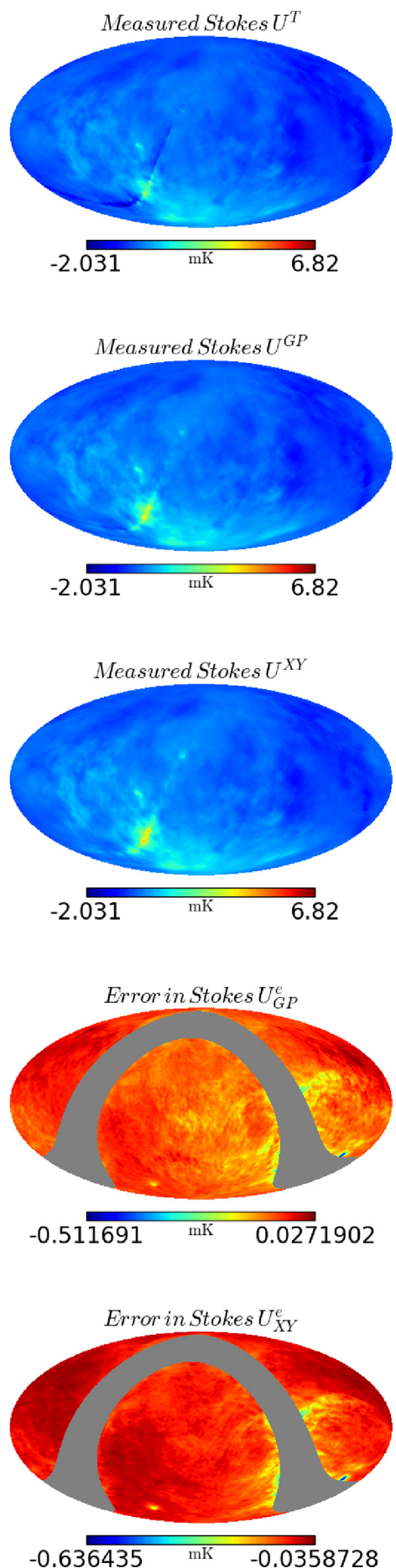

Figure 7. The first-row maps depict the measured foregrounds of Stokes $I, Q$, and $U$ for using the non-distorted fully polarized beams in Fig. 4(a) whilst the second and third rows represent the corrupted measured foregrounds due to gain and phase and dipole orientation errors introduced into the beams, respectively. The next two maps are the corresponding errors in $I, Q$, and $U$.

$21 \mathrm{~cm}$ brightness temperature at $z \approx 0.67$ produced from the $\mathrm{CRIME}^{14}$ fast-simulation software and described by Alonso, Ferreira \& Santos

${ }^{14}$ http: //intensitymapping.physics.ox.ac.uk/CRIME. html
(2014) is generated and then compared with the spectra plots of the Galactic foregrounds. The $\mathrm{H}$ I signal power on the right side of the plot is higher than $|Q+i U| \longrightarrow I$ at a multipole moment of $l=$ 100 , which is about 4 orders of magnitude greater at lower scales. This occurs when we do not correct the beam errors (i.e. gain, phase, and orientation) in Stokes $I$ at all. The fractional leakage of $\mid Q+$ 

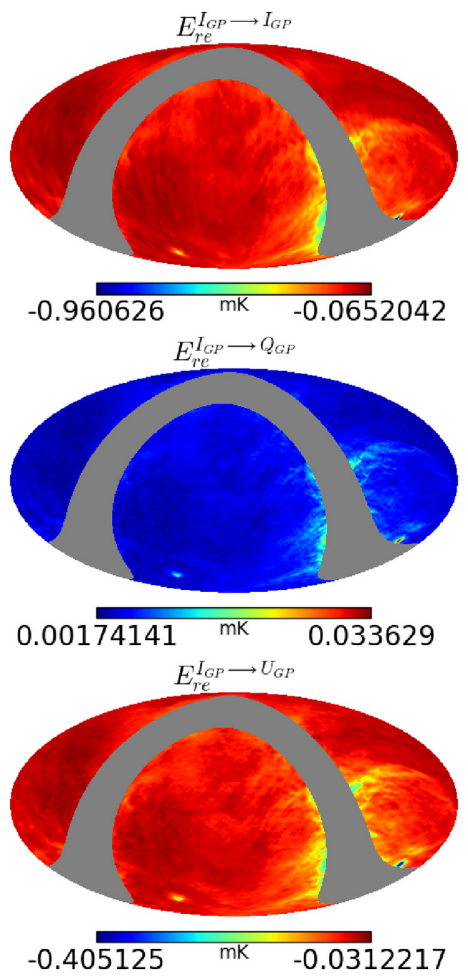
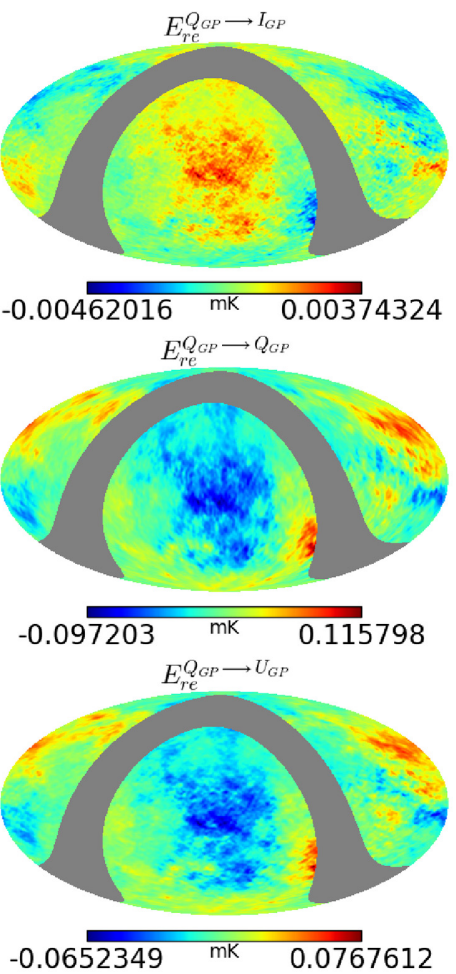
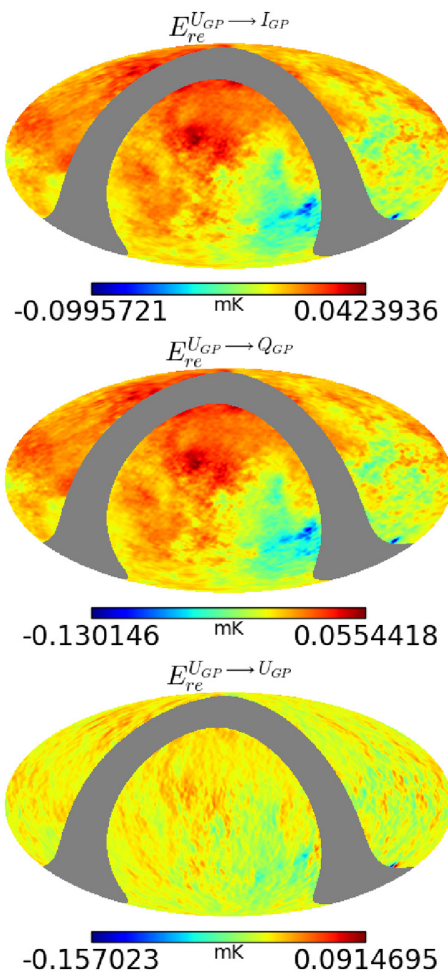

Figure 8. Systematic errors of full-sky maps produced by computing the relative error between the absolute of the convolved true sky maps and the corrupted sky maps due to gain and phase error beams.
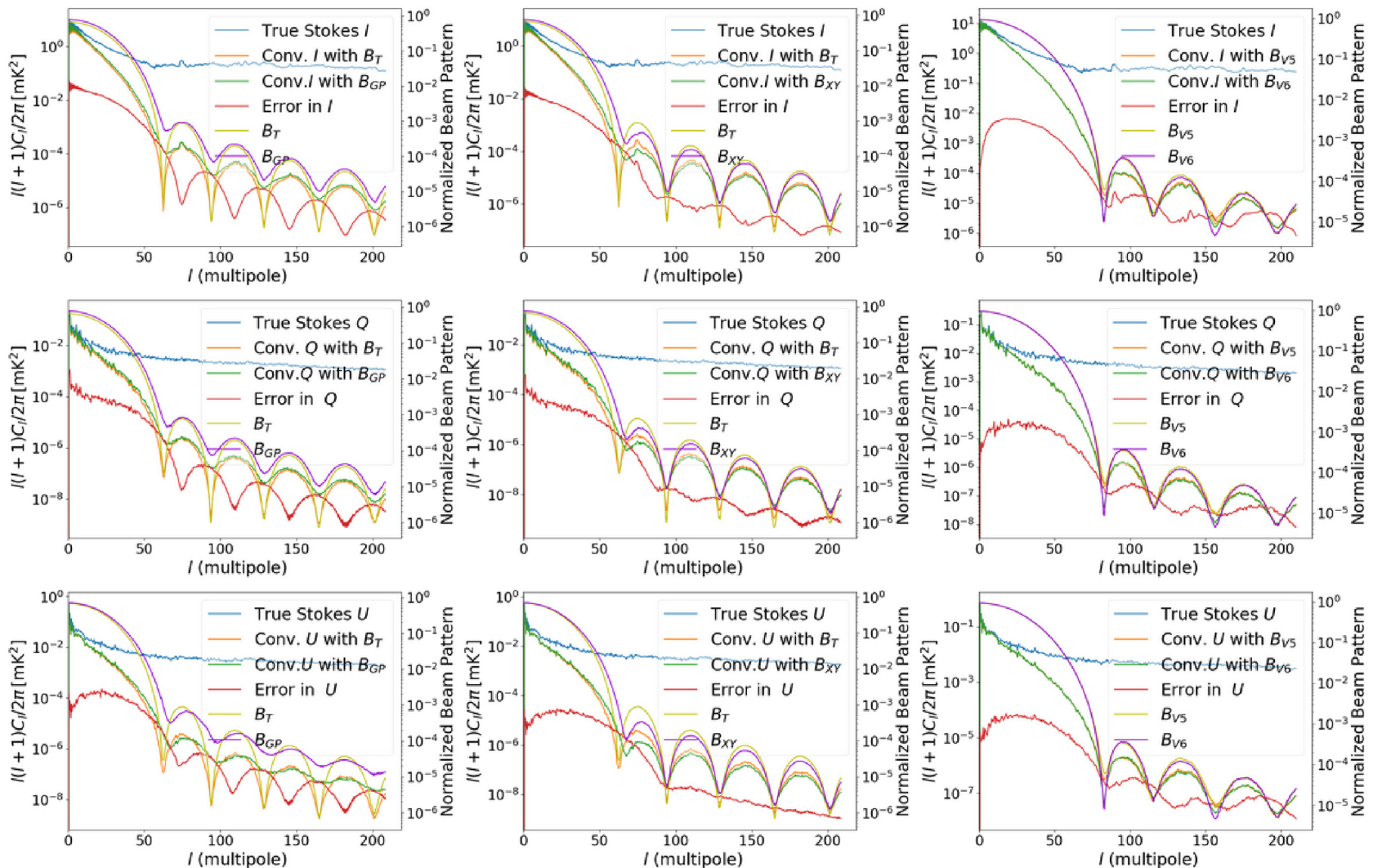

Figure 9. Convolved angular power spectra estimation of foreground maps. First row: Stokes $I$ spectra plots for using simulated beams and holographymeasured beams. Second row: Stokes $Q$ spectra plots for using simulated beams and holography-measured beams. Third row: Stokes $U$ spectra plots for using simulated beams and holography-measured beams. 

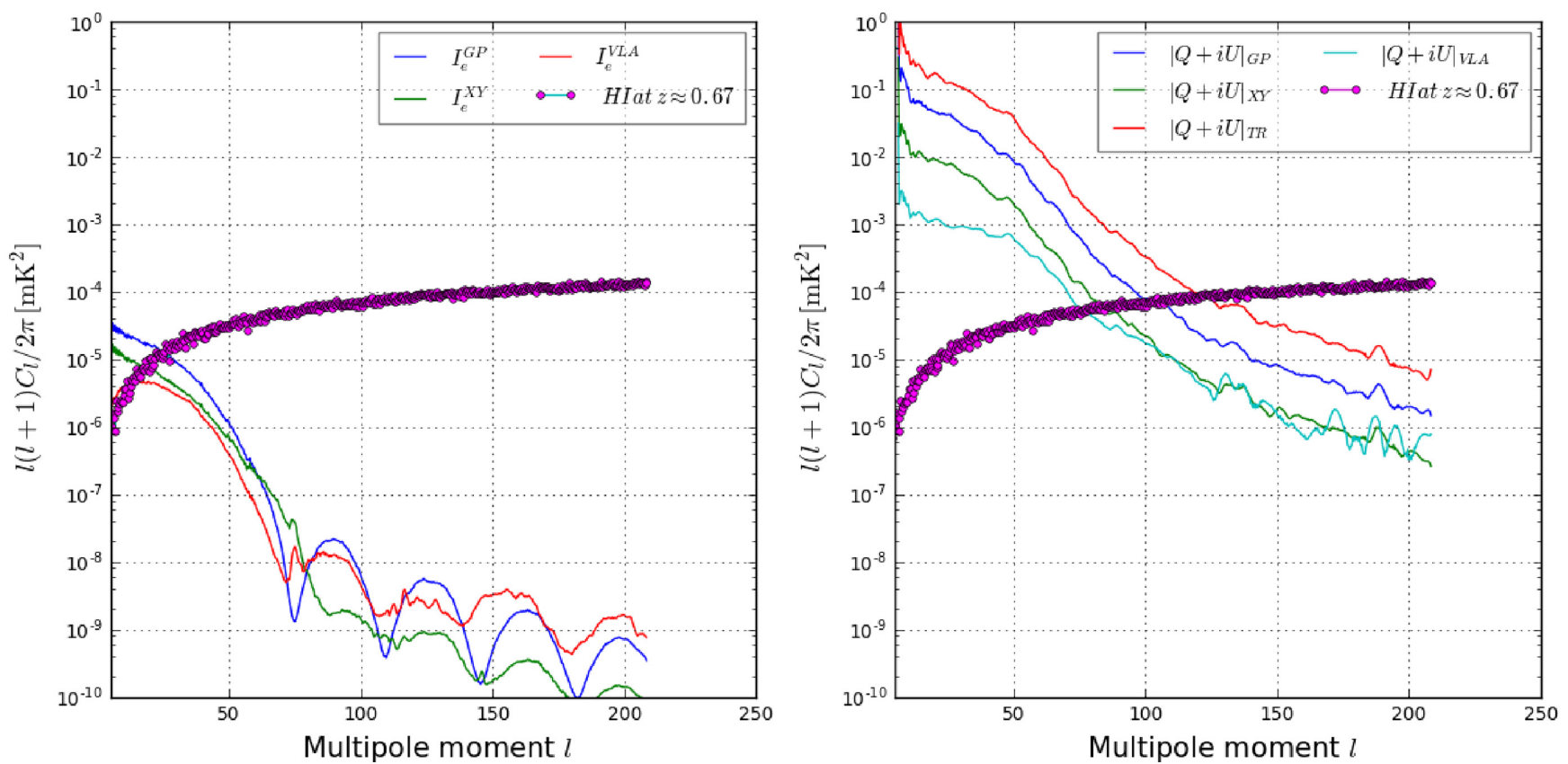

Figure 10. The spectra plots compare the effect of recovering the cosmological $21 \mathrm{~cm}$ signal by calibrating for the beam errors in Stokes $I$ to when there is no beam correction at all. The solid circular spectrum is the simulated $21 \mathrm{~cm}$ brightness temperature described by Alonso et al. (2014) at $z \approx 0.67$. Left-hand plot: Here, we show how to estimate the $21 \mathrm{~cm}$ signal when we correct the errors in Stokes $I$. Right-hand plot: We quantify the amount of leakages into Stokes $I$ when we do not perform any beam correction. The spectrum $\left(|Q+i U|_{T}\right)$, is the intrinsic leakage in $I$ when we adopt true modelled beams as shown in Fig. 4(a). The other plots $\left(|Q+i U|_{G P},|Q+i U|_{X Y},|Q+i U|_{\mathrm{VLA}}\right)$ are the leakages in $I$ when we use perturbed modelled beams (i.e. gain, phase, and main dish surface orientation errors) and holography-measured beams, respectively.

$i U \mid / I$ is computed to give $\approx 1.0$ per cent for the intrinsic case (i.e. $\left.|Q+i U|_{\mathrm{T}}\right)$ where a true model of the beam is known. The spectra plots reported in the other plot try to correct the columns that feed into Stokes $I$ (i.e. $Q \longrightarrow I, U \longrightarrow I$, and $I \longrightarrow I$ ) by assuming the corresponding beams (i.e. $m_{Q I}, m_{U I}$, and $m_{I I}$ ) are not known to the extent to which they have been assumed in this paper. In this case, the power spectrum of the $\mathrm{H}$ I signal can be observed at a multipole moment of $l=25$. We conclude that if the knowledge of the beam is of a similar quality than the one assumed in this paper, then we will be able to recover the cosmological $\mathrm{H}$ I signal without great problems and without further calibration on scales larger than $l=100$. However, this work suggests that if polarization calibration is performed correctly then results can be improved and we can recover scales above $l=25$. In either case, given the strength of the foregrounds in the Galactic Centre we will not be able to recover scales less than that (i.e. $l<25$ ).

The spectra plots in Fig. B3 evaluate the systematic effects of beam errors on Stokes $I, Q$, and $U$. These residuals are determined as a result of the respective differences between the distorted and non-distorted measured full-sky maps. We then compute the standard errors of these residual plots to estimate the uncertainties in the angular power spectra when modelled beams are assumed whilst the foreground maps are convolved with the measured beams (VLA holography beams). Table B1 in Appendix B shows the corresponding inaccuracies in the power spectrum estimation. For instance, the standard errors introduced in $Q \longrightarrow I$ are $\approx 0.015$ (due to gain and phase errors) and 0.014 (due to the main dish surface orientation errors). Also, those of $U \longrightarrow I$ are $\approx 0.005$ and 0.0045 , accordingly. The uncertainties in the spectra plots are a result of the inaccuracies on the surface of the modelled dish presented in Fig. 3.

\section{CONCLUSIONS}

The study introduces an application of the OSKAR software as a relatively cheap technique to produce realistic PBs and perturbations (using gain, phase, and main dish surface orientation errors) for IM experiments. These fully polarized modelled beams are then used to simulate the full-sky polarization maps by the method of convolution in order to compute the intensities of the diffuse Galactic foregrounds and determine the amount of signal that has seeped from linear polarization into total intensity. The simulation is repeated using the holography-measured beams and then compared with the modelled beams in order to estimate the error introduced in the power spectrum when modelled beams are used. The following are the key findings of the research:

(i) We use 80000 dipoles to model the distribution of the dishlike surface of the antenna. This produces $\approx 0.10$ per cent perturbed inaccuracies on the dish surface due to the random placement of the dipoles, which are not exactly phased up. The value of the perturbed inaccuracies will increase if the number of dipoles is $\ll 80000$.

(ii) The perturbed inaccuracies due to the imperfections in the nominal orientation of the dipoles introduce fractional errors of 0.08 for Stokes $I, 0.03$ for $Q$, and 0.07 for $U$ in the convolved power spectrum estimation. Note that these occur when we assume to use modelled beams whilst we convolve the foregrounds with measured beams.

(iii) Furthermore, if we construct a model of a beam and then carry out polarization rotation and calibration of the phase in order to correct the beam in Stokes $I$, then the power of the $\mathrm{H}$ I signal can be estimated at a multipole moment of $l=25$. But, if we don't do 
any correction at all for the beam, then the power spectrum of the $\mathrm{H}$ I signal is measured at a multipole moment of $l=100$. This makes the latter multipole moment be $\approx 4$ orders of magnitude higher than when we correct the error in the beam.

(iv) Finally, if a true model of the beam is assumed, then the intrinsic fractional leakage of $|Q+i U|_{T} \longrightarrow I$ is $\approx 1.0$ per cent.

In summary, the outputs of this research have shown how OSKAR can be used for beam pattern simulations and also introduce perturbations such as gain, phase, and surface orientation errors. Hence, with the fully polarized modelled beams produced from OSKAR and the convolution technique for foreground simulations, we can estimate the amount of foregrounds that have leaked from intensity into polarization and vice versa. Our future work is to implement a similar approach with MeerKAT holography-measured beams to investigate the effects on MeerKAT H I IM observation.

\section{ACKNOWLEDGEMENTS}

The authors acknowledge with profound gratitude full financial support from the South African Square Kilometre Array (SKA-SA) Project. The research of O. Smirnov is supported by the South African Research Chairs Initiative (SARChI) of the Department of Science and Technology and National Research Foundation. We also express our appreciation to the entire Radio Astronomy Techniques and Technologies (RATT) team for their immense contribution, particularly Dr. Griffin Foster. F.B. Abdalla acknowledges the support of the Royal Society via a University Reserach Fellowship (URF).

\section{REFERENCES}

Abitbol M. H., Hill J. C., Johnson B. R., 2016, MNRAS, 457, 1796

Alonso D., Ferreira P. G., Santos M. G., 2014, MNRAS, 444, 3183

Alonso D., Bull P., Ferreira P. G., Santos M. G., 2015, MNRAS, 447, 400

Bandura K. et al., 2014, in Stepp M. L., Gilmozzi R., Hall H. J., eds, Proc. SPIE Vol. 9145, Ground-Based and Airborne Telescopes V. SPIE, Bellingham, p. 914522

Bass M., DeCusatis C. M., Enoch J. M., Lakshminarayanan V., Li G., MacDonald C., Mahajan V. N., Van Stryland E., eds, 2009, Handbook of Optics: Geometrical and Physical Optics, Polarized Light, Components and Instruments. McGraw Hill Professional, New York, US

Battye R. A., Browne I. W. A., Dickinson C., Heron G., Maffei B., Pourtsidou A., 2013, MNRAS, 434, 1239

Bennett C. L. et al., 2013, ApJS, 208, 20

Bhatnagar S., Cornwell T. J., Golap K., Uson J. M., 2008a, A\&A, 487, 419

Booth R., Jonas J., 2012, African Skies, 16, 101

Born M., Wolf E., 1980, Principles of Optics Electromagnetic Theory of Propagation, Interference and Diffraction of Light. Pergamon Press, Oxford

Brentjens M. A., de Bruyn A. G., 2005, A\&A, 441, 1217

Brisken W., 2003, A Cassegrain Antenna Simulator, a Program for Cassegrain Antenna Modelling. http://packages.ubuntu.com/lucid/cass beam

de Oliveira-Costa A., Tegmark M., Gaensler B. M., Jonas J., Landecker T. L., Reich P., 2008a, MNRAS, 388, 247

de Vaate J. G. B., Faulkner A. J., 2012, Electromagnetics in Advanced Applications (ICEAA), p. 618-621

Decker F.-J., 1994, 6th Workshop on Beam Instrumentation, Beam Distributions Beyond RMS. Stanford University, Standford

Dulwich F., Mort B. J., Salvini S., Zarb Adami K., Jones M. E., 2009, Proceedings of Wide Field Astronomy \& Technology for the
Square Kilometre Array (SKADS 2009). p. 31, http://pos.sissa.it/cgibin/reader/conf.cgi?confid $=132$

Faulkner A. J., 2011, Dense Aperture Arrays for Square Kilometre Array, URSI General Assembly and Scientific Symposium XXX, 30, 1-4, IEEE, Istanbul, Turkey

Foley A. et al., 2016, MNRAS, 460, 1664

Geil P. M., Gaensler B. M., Wyithe J. S. B., 2011, MNRAS, 418, 516

Górski K. M., Hivon E., Banday A. J., Wandelt B. D., Hansen F. K., Reinecke M., Bartelmann M., 2005a, ApJ, 622, 759

Hamaker J. P., Bregman J. D., Sault R. J., 1996, A\&AS, 117, 137

Haslam C. G. T., Salter C. J., Stoffel H., Wilson W. E., 1982, A\&AS, 47, 1

Jelić V. et al., 2008, MNRAS, 389, 1319

Jelić V., Zaroubi S., Labropoulos P., Bernardi G., de Bruyn A. G., Koopmans L. V. E., 2010, MNRAS, 409, 1647

Jones R. C., 1942, J. Opt. Soc. Am., 32, 486

Jones R. C., 1948, J. Opt. Soc. Am., 38, 671

Kiyotomo I., 2014, Prog. Theor. Exp. Phys., 06

Kogut A. et al., 2007, ApJ, 665, 355

Krachmalnicoff N., Baccigalupi C., Aumont J., Bersanelli M., Mennella A., 2016, A\&A, 588, A65

La Porta L., Burigana C., Reich W., Reich P., 2008, A\&A, 479, 641

Lewis A., Challinor A., 2007, Phys. Rev. D, 76, 083005

Loeb A., Wyithe J. S. B., 2008a, Phys. Rev. Lett., 100, 161301

Masui K. W., McDonald P., Pen U.-L., 2010, Phys. Rev. D, 81, 103527

Masui K. W. et al., 2013, ApJ, 763, L20

Miville-Deschênes M.-A., Ysard N., Lavabre A., Ponthieu N., Macías-Pérez J. F., Aumont J., Bernard J. P., 2008a, A\&A, 490, 1093

Newburgh L. B. et al., 2014, inStepp M. L., Gilmozzi R., Hall H. J., eds, Proc. SPIE Vol. 9145, Ground-Based and Airborne Telescopes V. SPIE Bellingham, p. 91454V

Newburgh L. B. et al., 2016, inHall H. N., Gilmozzi R., Marshall H. K., eds, Proc. SPIE Vol. 9906, Ground-Based and Airborne Telescopes VI. SPIE, Bellingham, p. $99065 \mathrm{X}$

Oppermann N. et al., 2012, A\&A, 542, A93

Perley R. et al., 2015, , Optimizing VLA Antenna Performance, EVLA Memo XXX, NRAO.

Peterson J. B. et al., 2009, in Astro2010: The Astronomy and Astrophysics Decadal Survey. National Academy of Sciences USA, p. 234

Planck Collaboration VIII, 2016a, A\&A, 594, A8

Planck Collaboration VIII, 2016b, A\&A, 594, A10

Pogany T. K., Nadarajah S., 2009, Research Report 18, On the Characteristic Function of the Generalized Normal Distribution. Probability and Statistics Group School of Mathematics, the University of Manchester, Manchester

Saha R., Jain P., Souradeep T., 2006, ApJ, 645, L89

Santiago B. X., Strauss M. A., Lahav O., Davis M., Dressler A., Huchra J. P., 1995, ApJ, 446, 457

Santiago B. X., Strauss M. A., Lahav O., Davis M., Dressler A., Huchra J. P., 1996, ApJ, 461, 38

Santos M. G., Cooray A., Knox L., 2005, ApJ, 625, 575

Santos M. et al., 2015, PoS AASKA14, p. 19, Giardini Naxos, Italy

Schramm D. N., Galeotti P., eds, 1997, Generation of Cosmological LargeScale Structure, Kluwer Academic, Dordrecht

Shaw J. R., Sigurdson K., Pen U.-L., Stebbins A., Sitwell M., 2014, ApJ, 781,57

Shaw J. R., Sigurdson K., Sitwell M., Stebbins A., Pen U.-L., 2015, Phys. Rev. D, 91, 083514

Smirnov O. M., 2011, A\&A, 527, A106

Souradeep T., Saha R., Jain P., 2006, New Astron.Rev., 50, 854

Testori J. C., Reich P., Reich W., 2008, A\&A, 484, 733

The Polarbear Collaboration: , Ade P. A. R. The Polarbear Collaboration: et al., 2014, ApJ, 794, 171

Trott C. M., Wayth R. B., Tingay S. J., 2012, ApJ, 757, 101

Tucci M., Carretti E., Cecchini S., Nicastro L., Fabbri R., Gaensler B. M., Dickey J. M., McClure-Griffiths N. M., 2002, ApJ, 579, 607 
van Haarlem M. P. et al., 2013, A\&A, 556, A2

Varshalovich D., Moskalev A., Khersonskiǔ V., 1988, Quantum Theory of Angular Momentum: Irreducible Tensors, Spherical Harmonics, Vector Coupling Coefficients, 3nj Symbols. World Scientific Pub. http://books. google.ca/books?id=zPxlQgAACAAJ

White M., 1998, Phys. Rev. D, 57, 5273

Wijnholds S., van der Tol S., Nijboer R., van der Veen A.-J., 2010, IEEE Signal Process. Mag., 27, 30

Wolleben M., Landecker T. L., Reich W., Wielebinski R., 2006, A\&A, 448, 411

Wolleben M. et al., 2009, in Strassmeier K. G., Kosovichev A. G., Beckman J. E., eds, Proc. IAU Symp. Vol. 259, Cosmic Magnetic Fields: From Planets, to Stars and Galaxies. Kluwer, Dordrecht, p. 89

Wolz L., Abdalla F. B., Blake C., Shaw J. R., Chapman E., Rawlings S., 2014, MNRAS, 441, 3271

Wolz L. et al., 2015, PoS AASKA14, p. 35, Giardini Naxos, Italy

Woudt P. A., Fender R. P., Armstrong R. P., Carignan C., 2013, South Afr. J. Sci., 109, 01

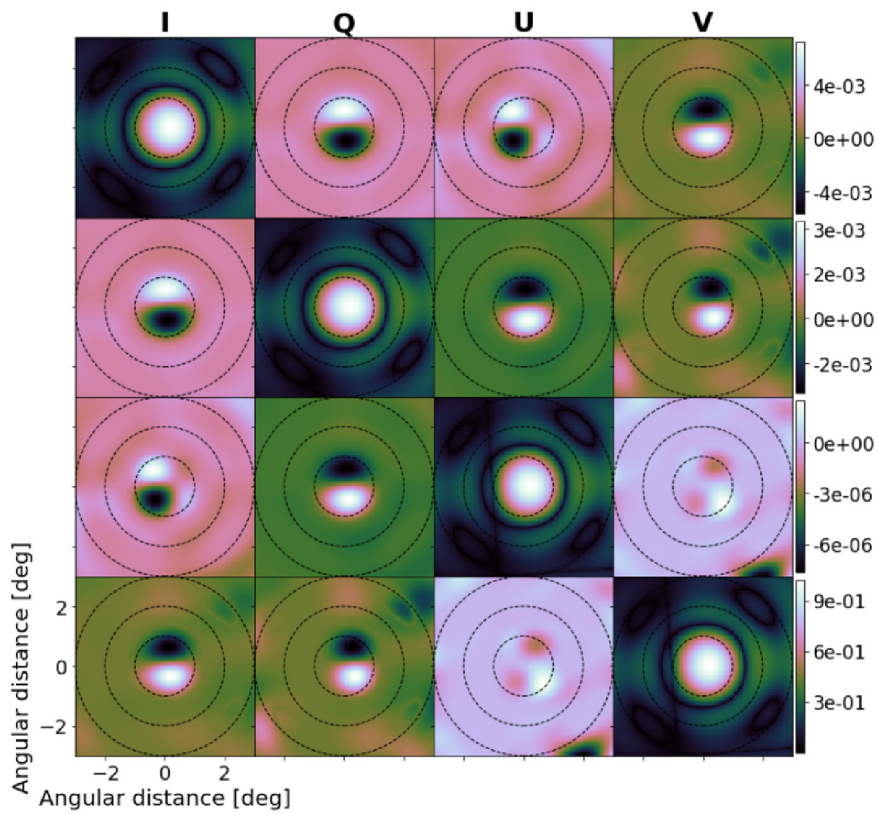

(a)
Wrobel J. M., Walker R. C., 1999, in Taylor G. B., Carilli C. L., Perley R. A., eds, ASP Conf. Ser. Vol. 180, Synthesis Imaging in Radio Astronomy II. Astron Soc. Pac., San Francisco, p. 171

\section{APPENDIX A: MODELLED AND MEASURED BEAMS}

The Mueller matrix representations in Fig. A1 show the different perturbation methods (i.e. gain, phase, and surface orientation errors) used to corrupt the OSKAR beam model. Note that the distortions in Fig. A1 are clearly visible at the upper and lower diagonals of the beams. These perturbed beams are then compared with the errors produced from 'real' measured beams of the VLA in Fig. A2. The PBs presented in Fig. A2 are taken from two different stations (i.e. antennas 5 and 6) whose fractional differences (refer to Fig. 4d) are relatively higher than those of any other pair of stations.

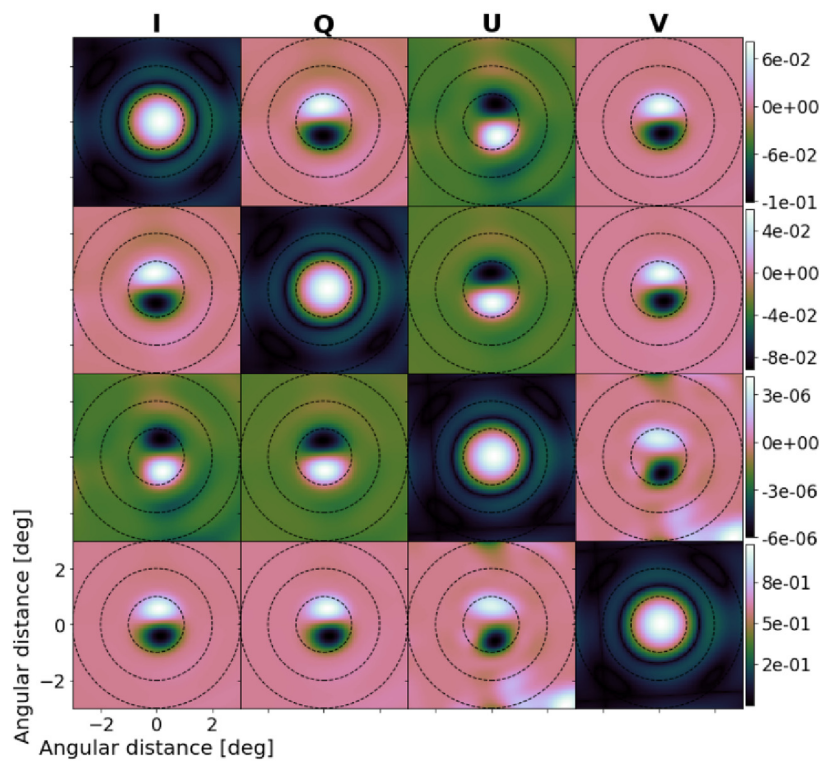

(b)

Figure A1. Fully polarized distorted primary beams of KAT-7. (a) Due to gain and phase errors. (b) Due to dipole orientation errors. 


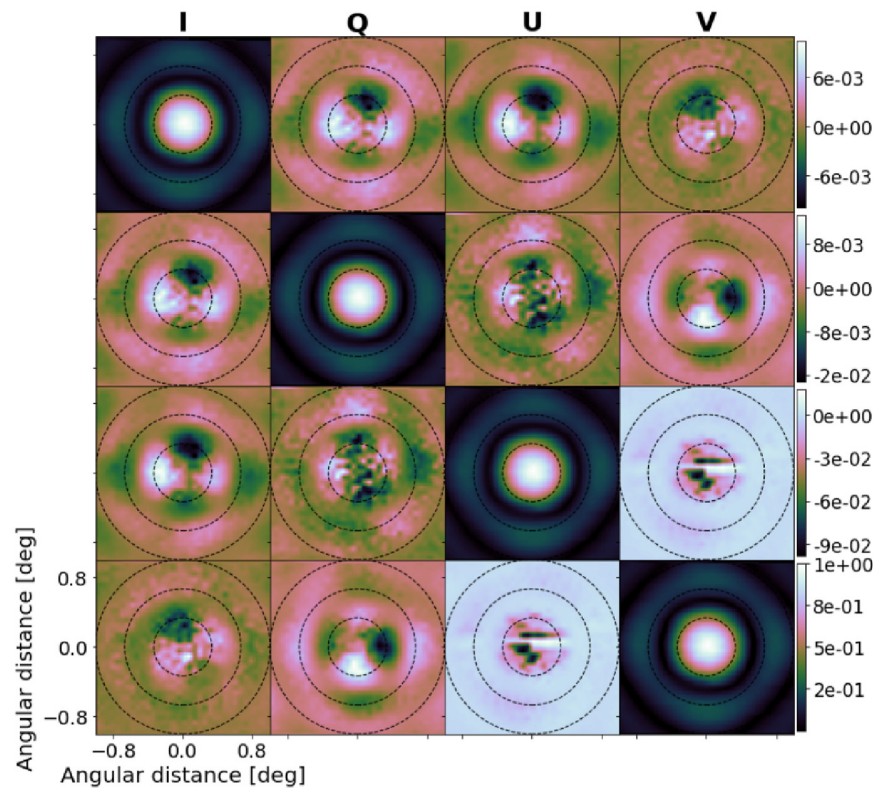

(a)

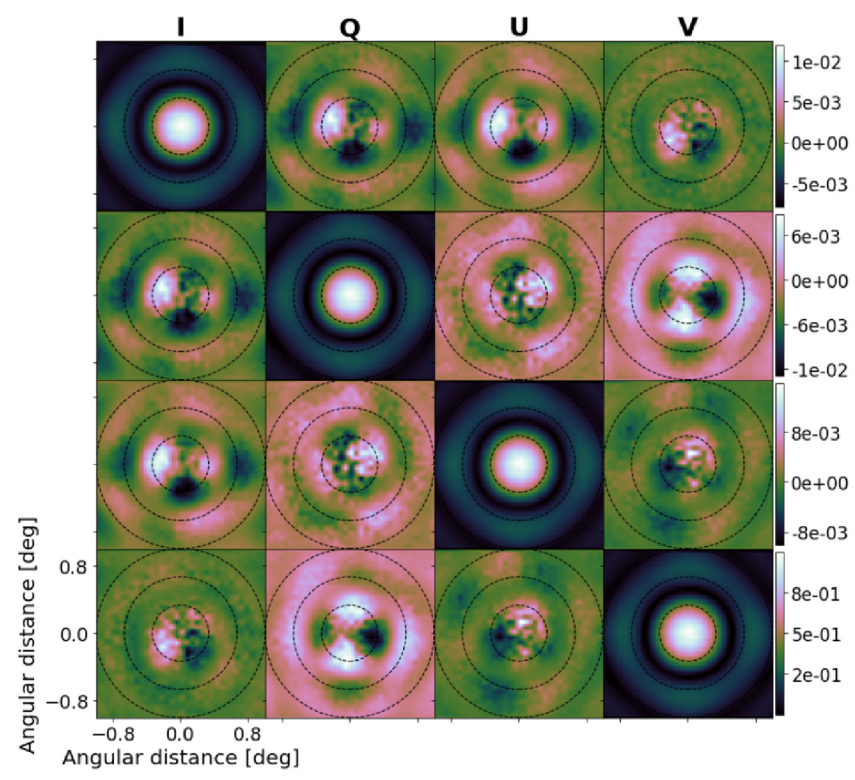

(b)

Figure A2. $1 \mathrm{GHz}$ holography-measured Mueller beams of VLA. (a) Antenna 5. (b) Antenna 6.

\section{APPENDIX B: MEASURED FULL-SKY MAPS}

Figs B1 and B2 represent the respective systematic error maps and the overall full-sky convolved maps simulated with the holographymeasured beams of the VLA in Fig. A2. The latter maps are used to produce the convolved power spectrum of the VLA as presented in Fig. 9.
Table B1 displays the errors recorded in the angular power spectrum estimation when modelled beams are assumed, whilst the foregrounds are actually convolved with real measured beams. These errors are tabulated from the absolute differences of the standard errors reported in Fig. B3. 


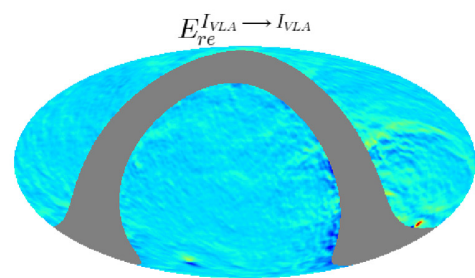

$\begin{array}{lll}-0.11208 & \mathrm{mK} & 0.224211\end{array}$
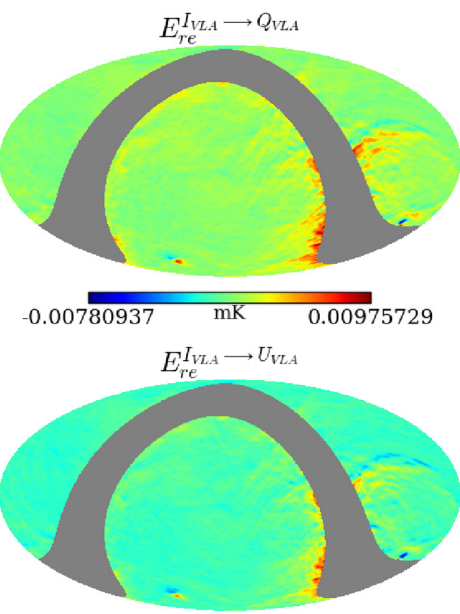

$\begin{array}{lll}0.0128719 & \mathrm{mK} & 0.0244106\end{array}$
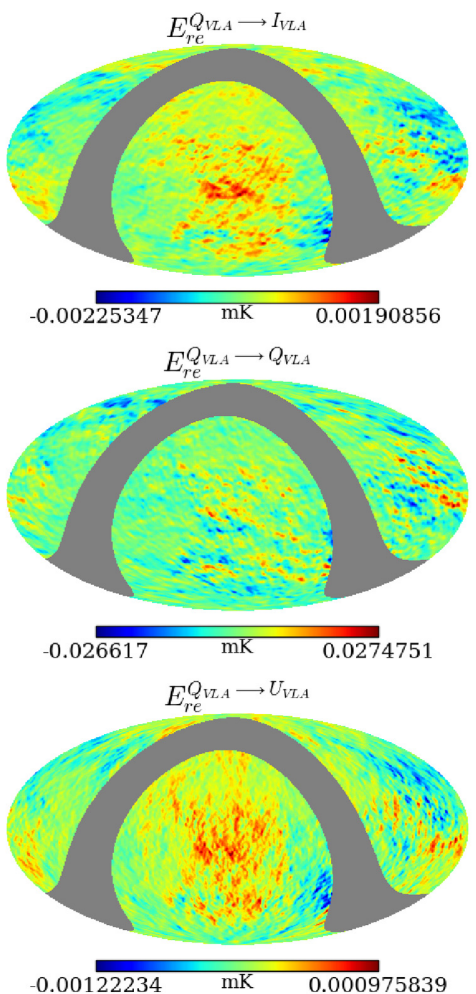
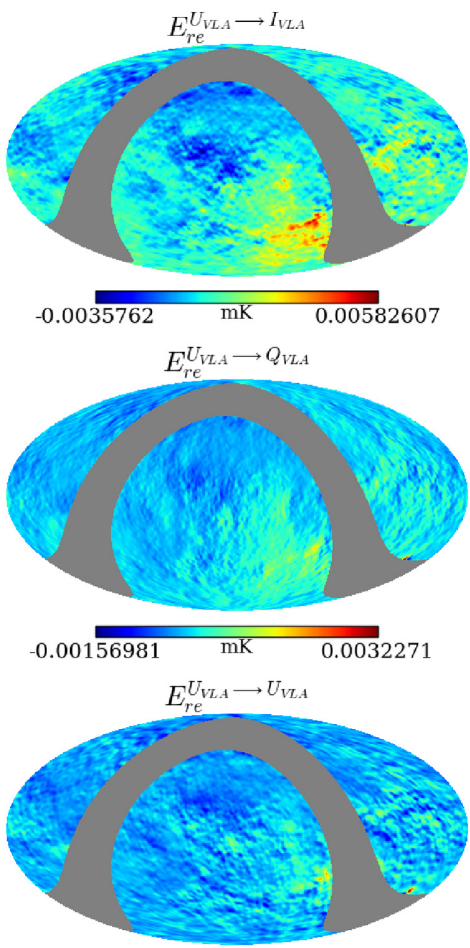

$\begin{array}{lll}0.0249898 & \mathrm{mK} & 0.0605507\end{array}$

Figure B1. Systematic differences of the full-sky polarization maps produced by computing the relative error between the convolved sky using VLA PBs in Fig. A2.
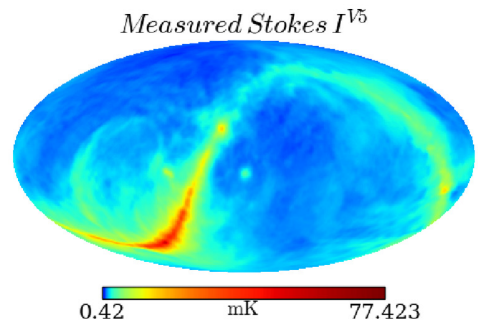

Measured Stokes $I^{\text {V6 }}$
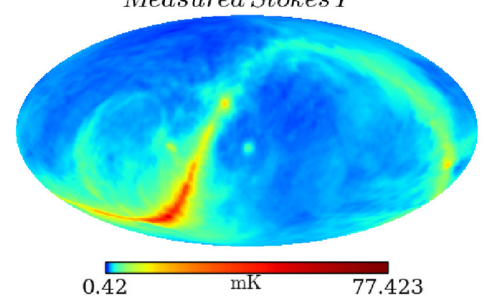

Error in Stokes $I_{V L A}^{e}$

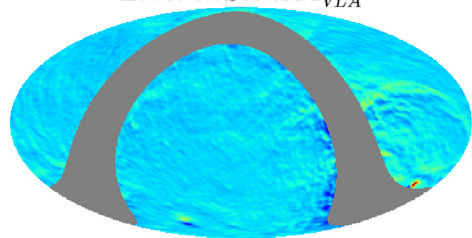

$\begin{array}{lll}-0.106695 \quad \mathrm{mK} & 0.221362\end{array}$
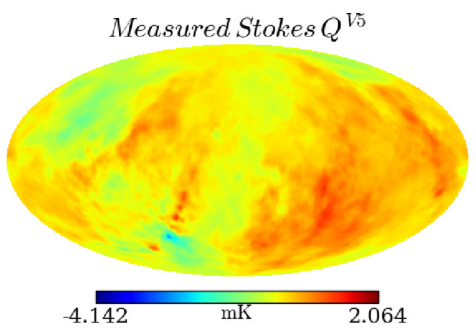

Measured Stokes $Q^{16}$
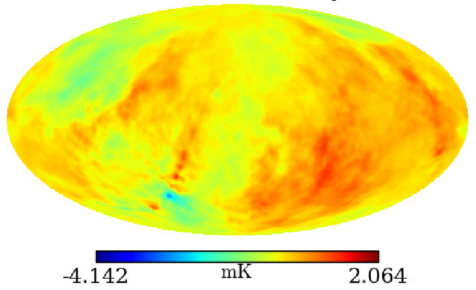

Error in Stokes $Q_{V L A}^{e}$

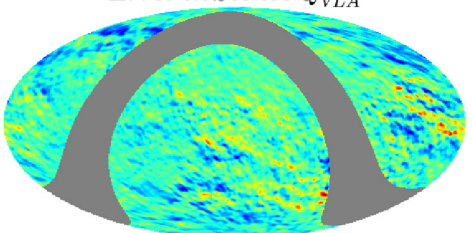

$\begin{array}{lll}-0.0204477 & \mathrm{mK} & \mathbf{0 . 0 3 1 5 0 6 2}\end{array}$

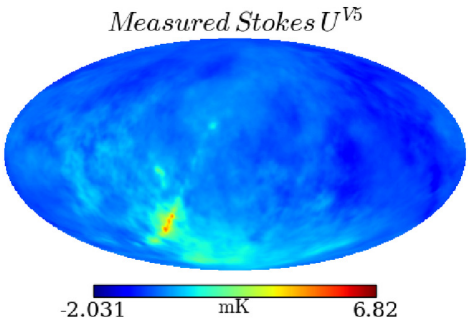

Measured Stokes $U^{\text {V6 }}$

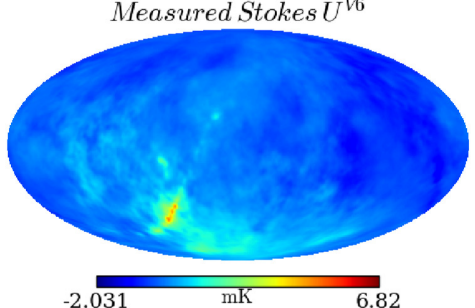

Error in Stokes $U_{V L A}^{e}$

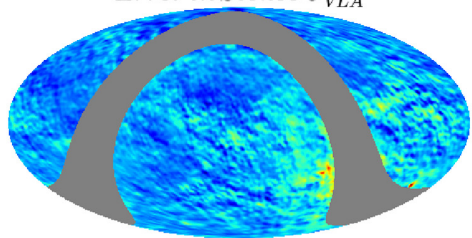

$\begin{array}{lll}-0.0165961 & \mathrm{mK} & 0.0554803\end{array}$

Figure B2. Measured Stokes $I, Q$, and $U$ for holography-measured beams of the VLA with corresponding errors terms. 
Table B1. Error introduced in the power spectrum estimation.

\begin{tabular}{lccccccccc}
\hline & & \multicolumn{2}{c}{$\boldsymbol{I}$} & & & & & & Total \\
& $G P$ & $X Y$ & $G P$ & $X Y$ & $G P$ & $X Y$ & $G P$ & $X Y$ \\
\hline$I$ & 0.0640 & 0.0640 & 0.0151 & 0.0137 & 0.0050 & 0.0045 & $\mathbf{0 . 0 8 4 1}$ & $\mathbf{0 . 0 8 2 2}$ \\
$Q$ & 0.0010 & 0.0008 & 0.0221 & 0.0224 & 0.0007 & 0.0055 & $\mathbf{0 . 0 2 3 8}$ & $\mathbf{0 . 0 2 8 7}$ \\
$U$ & 0.0007 & 0.0007 & 0.0194 & 0.0341 & 0.0354 & 0.0362 & $\mathbf{0 . 0 5 5 5}$ & $\mathbf{0 . 0 7 1 0}$ \\
\hline
\end{tabular}
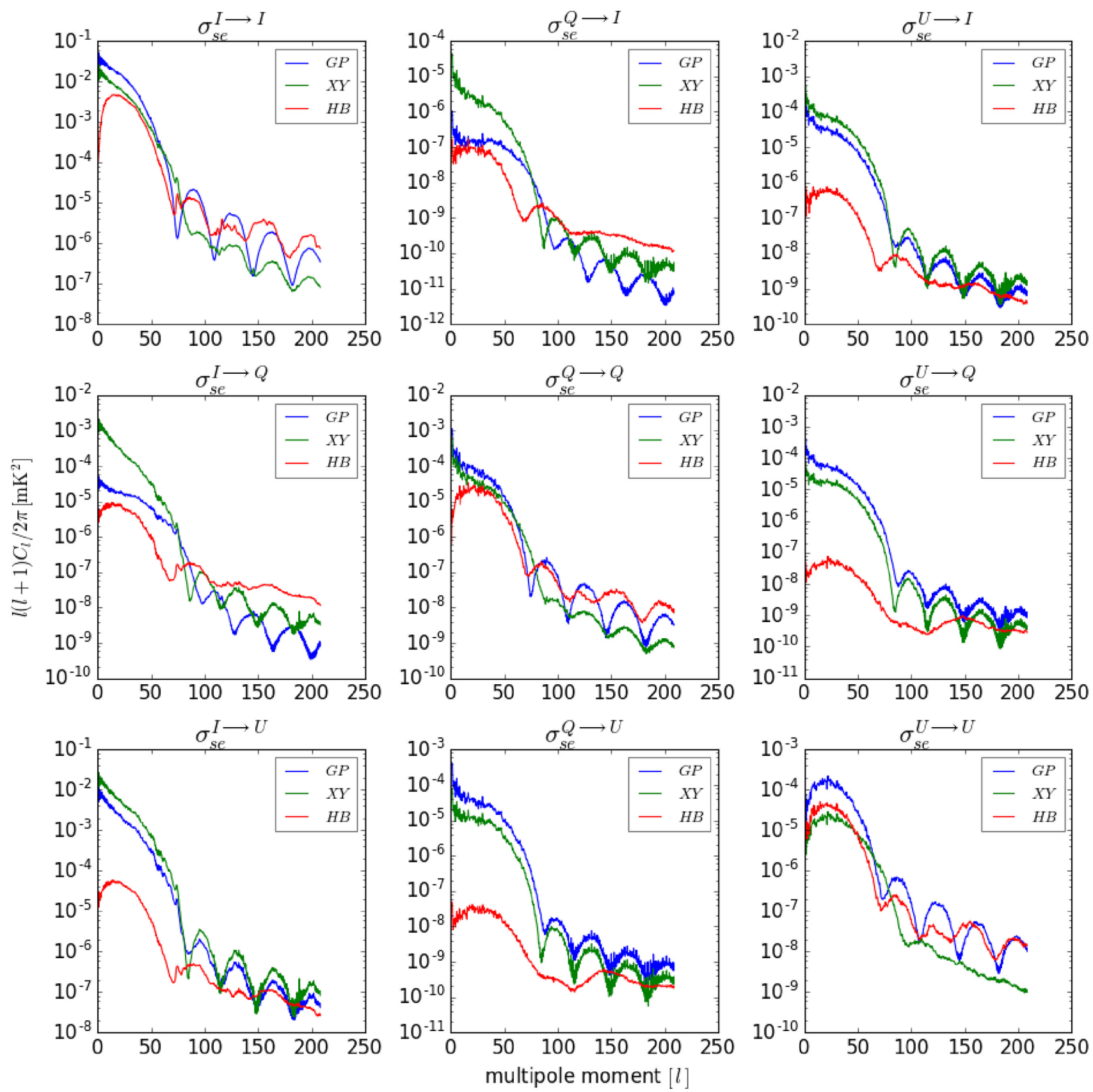

Figure B3. These are the spectra plots of the systematic errors as shown in Fig. 8. The notations $G P$ and $X Y$ in the legends denote the residuals for gain-phase and surface orientation errors in the OSKAR beams; $H B$ depicts the errors in the holography beams. These errors are then used to estimate the imperfections in the simulation by computing the expected value of the standard deviations of the sampling distributions of the residual maps to produce Table B1.

This paper has been typeset from a $\mathrm{T}_{\mathrm{E}} \mathrm{X} / \mathrm{L} \mathrm{T}_{\mathrm{E}} \mathrm{X}$ file prepared by the author. 